\title{
INTELLIGENT SERVICING STRATEGY FOR AN ONLINE-TO-OFFLINE (O2O) SUPPLY CHAIN UNDER DEMAND VARIABILITY AND CONTROLLABLE LEAD TIME
}

\author{
Seok-Beom Choi $^{1}{ }^{\circledR}$, Bikash Koli Dey ${ }^{2}$,, Sung Jun Kim³ and Biswajit SarkaR ${ }^{3,4, *}$
}

\begin{abstract}
With the advancement of technologies, industries tries to adopt the advantages of the technology. Customers are busy in their daily life, and the online platform is the best option for retail, whereas traditional customers still prefer to visit the retail shop. Few customers choose the product online but buy it offline or vi ce-versa. Ow ing to all th ose ci rcumstances, cu rrent st udy fo cuses on an intelligent dual channel (online-to-offline) st rategy in in dustry to ar range the op timal se rvices for customers. The selling price of the product vary with different $\mathrm{c}$ hannel, w hich h elps t o determine the demand of product for entire supply chain. Two important factors, backorder and lead-time are examined precisely through marginal value which helps to arrange optimal service and calculate the exact profit. The profit for a centralized and decentralized case are computed for both the players. Some propositions are developed to prove the global optimality. Numerical results prove that a centralized case provides $7.77 \%$ better profit than a decentralized c ase due to b onding between the players.
\end{abstract}

Mathematics Subject Classification. 90B05, 90B06.

Received December 22, 2021. Accepted February 10, 2022.

\section{INTRODUCTION}

In those days, due to implementation of information technology and uses of different smart delivery policy, world was witness of huge changes in supply chain industry. Uses of technology and internet growing rapidly, and customers are more familiar with online order system. Though, some customers still believe in traditional offline marketing. Nowadays, most of the people want to order product through online channel, whereas there are few customers, who believe in traditional offline channel. They visit particular store and choose their product physically. The main target of any industry is to optimize their cost via different policy Sarkar and Chung [50] and the most important issue for optimizing the profit or cost is customer satisfaction. To satisfy the customers, nowadays different strategies were utilized by the industry managers. However, customers are always satisfied

Keywords. O2O retailing, variable lead time, supply chain management, sales-price-dependent demand, backorder.

1 Institute for Social Science Research, Jeju National University, Jeju-do 63243, South Korea.

2 Department of Industrial \& Data Engineering, Hongik University, Wausan-ro 94, Mapo-Gu, Seoul 04066, South Korea.

3 Department of Industrial Engineering, Yonsei University, 50 Yonsei-ro, Sinchon-dong, Seadaemun-gu, Seoul 03722,

South Korea.

4 Center for Transdisciplinary Research (CFTR), Saveetha Dental College, Saveetha Institute of Medical and Technical Sciences, Saveetha University, 162, Poonamallee High Road, Velappanchavadi, Chennai 600077, Tamil Nadu, India.

*Corresponding author: bsbiswajitsarkar@gmail.com 


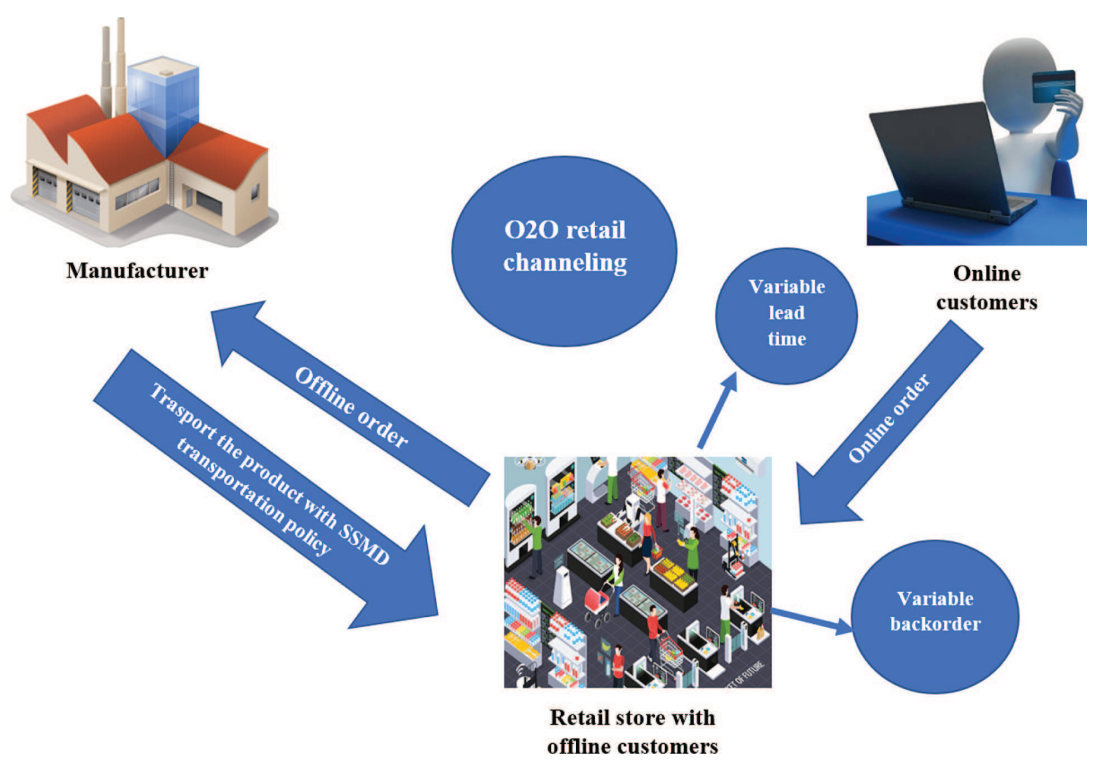

Figure 1. Graphical illustration of $\mathrm{O} 2 \mathrm{O}$ retail dual channel.

through the good service and timely service provided by the particular company. Thus, upgradation of service level is one of the most effective way to enhance the system profit. In this regards, researchers are always trying to develop some new strategy such that company can provide best service to their customers as well as optimize total system profit.

It is well known to all that time is money and anyone don't want to waste time. To provide better service and exact lead time is very much essential. Moreover, through the exact rate of backorder, company can take exact decision about production system. Thus, exact lead time and exact backorder always provide better service as well as enhance supply chain's profit [33]. Simultaneously, price of any product is one of the decision maker to find the demand of any product, less price generally attracts the customers more and increase the demand of any product. The cost for different parameters like holding, setup and ordering can not always be fixed [50]. Setup and ordering cost of production system can be controlled with investment [7]. Now, what are the motivation of this current study? is describe as follows:

(i) There are several supply chain or online-to-offline channel model in literature [23,51], however there are few models [52] where, demand varies with both channel price.

(ii) Lead time of SC can be controlled through investing some amount in production system [38]. However, calculation of exact lead time and exact backorder with the help of marginal value is very rare [33].

(iii) Several SCM were constructed along with variable setup cost or improved process quality [7]. However, variable ordering cost and reduction of ordering cost through continuous investment are very rare [52].

Thus, in this current study a single-retailer, single-manufacturer online-to-offline advanced supply chain model for a single item was presented under the consideration of online and offline demand, where both channel demands depend on selling price. Moreover, exact lead time and backorder are calculated through marginal value, which helps to obtain exact cost or profit of the system along with some continuous investments which directly reduce the ordering cost for the retailer and increase the reliability of the production process for the manufacturer under uncertain environment. Thus, this is the first try where all those research gap was full filled along with optimized system profit for the advanced dual channel system. A graphical representation of $\mathrm{O} 2 \mathrm{O}$ dual channel is presented in Figure 1. 
TABLE 1. Novelty of the study with existing literature.

\begin{tabular}{llllll}
\hline \hline Author(s) & Supply chain & Strategy & Lead time & Backorder & Demand \\
\hline Sarkar and Chung [50] & TE & Cen. & DFA & NA & Con. \\
Li [33] & Normal & Cen. & Var. & Planned & Con. \\
Dey et al. $[12]$ & TE & Cen. & NA & NA & AD \\
Sarkar et al. $[52]$ & O2O & Cen. & DFA & Planned & OOPD \\
Mashud et al. $[38]$ & Inv. & Trade-credit & NA & NA & PD \\
Guchhait and Sarkar [21] & Normal & Cen. & Var. & Partial & Con. \\
Ji et al. $[26]$ & O2O & Cen. & NA & NA & Con. \\
Sett et al. $[58]$ & O2O & Crisp & Normal & Planned & PD \\
This study & O2O & Cen. \& & MVL \& & PE & OOPD \\
& & Dcen. & VV & & \\
\hline
\end{tabular}

Notes. NA - Not Applicable, O2O - O2O retailing, Int. - Integrated supply chain, TE - Two echelon, DFA - Distribution free approch, Cen - Centralize, Dcen. - Decentralize, PE - Planned \& exact, PD - Price dependent, VV - Variable variance, Con. - Constant, Var. - Variable, APD - Advertisement \& Price dependent, OOPD - Online \& offline price dependent, and MVL - Marginal value with respect to lead time.

The current study structured as: The next Section 2 contains the gap of literature and the previous study in this direction in terms of literature review. The main problem, which was solved in this study is describe in the Section 3 along with notations and assumption of this study. Section 4, contains the formulation of model for manufacturer and retailer. The solution methodology for centralize and decentralize case is illustrated in Section 5. To show the applicability of this study, some examples based on parametric value are proposed in Section 6. Change in total profit along with key parameters is calculated in Section 7. Findings, limitations and concluding remarks with some future extensions are described in the Conclusion Section 8.

\section{ILLUSTRATION OF EXISTING RESEARCH WORK}

The illustration of the existing literature, gaps in the literature, and novelty of the present study compared to the existing research are elaborately discussed through the following subsections. The gaps in the literature and novelty of the current study compared to existing study tabulated in Table 1.

\subsection{Dual channel supply chain management (SCM)}

SCM is the administration of the stream of products and administrations and incorporates all forms that change crude materials into last items. It includes the dynamic streamlining of a business's supply-side exercises to maximize client esteem and pick up a competitive advantage within the commercial center. A theoretical framework for strategy development to introduce sustainable SCM was discussed by Govindan [20]. They incorporated the concept of sustainability along with lean, resilient and green SCM. The disruption of transportation within the players discussed by Clemons and Slotnick [10]. They have to show that the viability of information exchange includes a noteworthy affect on benefit. Bhuniya et al. [6] proposed an imperfect production-based SCM through quality improvement, and setup cost reduction strategy. Sarkar et al. [53] developed a sustainable SCM of a highly perishable fixed and lifetime base product. Bhuniya et al. [7] proposed smart production process under a SCM through rework, restoration strategy, and maintenance policy.

A convenient ways to effective business today is online-to-offline business strategy. The most important thing is the huge combination of choices. However, O2O supply chain model is not well known in the academic world and e-commerce companies may have different ideas about $\mathrm{O} 2 \mathrm{O}$ business model.

$\mathrm{O} 2 \mathrm{O} \mathrm{SCM}$, a modern generation of commerce model is put forward by a few neoteric exertions. In any case, the $\mathrm{O} 2 \mathrm{O}$ supply chain model isn't well known within the scholastic world and e-commerce companies may have diverse thoughts around $\mathrm{O} 2 \mathrm{O}$ trade model. An $\mathrm{O} 2 \mathrm{O}$ retail SC inside the setting of low-carbon environment 
demonstrate is created by Ji et al. [26] to compare the impacts and the benchmarks components on the firm's benefits, choices, and social welfare. A system for assessment of supply chain synthesis by contracts beneath $\mathrm{O} 2 \mathrm{O}$ environment model was developed by Govindan and Malomfalean [19]. This paper builds up a contrast based on $\mathrm{O} 2 \mathrm{O}$ direction of two types of demand. Li et al. [34] inspected a pleasing advancing technique within the $\mathrm{O} 2 \mathrm{O} \mathrm{SC}$ made of a merchant and a web organize administrator. This model considered consider pleasant advancing blueprints for this $\mathrm{O} 2 \mathrm{O} \mathrm{SC}$ and look at three models.

An $\mathrm{O} 2 \mathrm{O}$ policy and self-scheduling distribution capacity of grocery stores model is introduced by $\mathrm{He}$ et al. [24]. They developed appropriation obligations for the O2O stage to adjust its and the conveyance platform's goals. In the same year an $\mathrm{O} 2 \mathrm{O}$ retailing strategy under price, quality, and service dependent demand was constructed by Sett et al. [58]. An $\mathrm{O} 2 \mathrm{O}$ retail channel can be more profitable due to the influence of the social network [47]. Pei et al. [45] introduced an imaginative consistency instrument to reduce channel fighting and a producer advertised to offline customers makes a difference diminish the $\mathrm{O} 2 \mathrm{O}$ supply chain. A low-carbon tourism $\mathrm{O} 2 \mathrm{O}$ SCM is introduced by Ma et al. [36]. The aim of this model shows that the benevolent inclination can at the same time advance the economical improvement of the low-carbon tourism SC and accomplish SC coordination. Sarkar et al. [52] created a two-echelon SCM for the offline-to-online framework beneath the thought of dissemination free approaches and optimize the framework benefit.

Nowadays, O2O business strategy strongly depends on supply chain management. The selling cost of the items is connected with the $\mathrm{O} 2 \mathrm{O}$ supply chain administration. The items demand much depends on the offering cost for the $\mathrm{O} 2 \mathrm{O}$ supply chain. Thus, we are moving this discussion forward in the next paragraph.

\subsection{Selling price dependent demand}

Price of the product always takes a major part for any business industry. Price for the items was always very crucial for any supply chain [54]. Different policies are required to fulfill the goals for sustainable development [2]. To rectify the waste for a supply chain system to enhance the circular economy and sustainable development is very essential in today's business sector [55]. Not only price, quality of the product had also a great impact for determine the demand [27]. Several studies were conducted in literature, in which retail price takes a major role to take several major decisions. To develop an intelligent system, a huge amount will be needed in setup, which can be reduced through some discrete investment, and profit of the integrated system can be optimized by taking decision on the price of the product [38]. Guchhait and Sarkar [21] calculated the emitted carbon during transportation to make a sustainable SC. Uncertain market condition can be solve by using the novel concept of fuzzy set theory. In this direction a modified optimization technique for quadrilateral fuzzy number was invented by Kumar et al. [30]. In the similar direction a production model with variable lead time under fuzzy demand was proposed by Mahapatra et al. [37]. A close loop supply chain under uncertain fuzzy $T$ environment was developed by Garai et al. [17]. They also discussed about the subsidy policy for the biofuel plants. Under the uncertain environment some robust optimization technique for animal-fat based biodiesel was proposed by Habib et al. [22]. Some models were formulated under price sensitive demand pattern along with some new optimization technique. Not only selling price, quality of the products take a major role to take several major decision on demand [13]. The quality of the product can be major through an intelligent autonomation policy was introduced by Dey et al. [13]. Autonomation inspection can perform error-free inspection. Price along with stock-level of the product is crucial for industry Pando et al. [44]. In the similar direction, Xu et al. [61] proposed a dual channel SCM, where online demand takes a major part to make decisions. Chaudhuri et al. [9] considered price and service dependent demand for their manufacturing model. Recently, Sarkar et al. [52] developed a dual channel SCM, under the consideration prices dependent demand. They used distribution free approach for the lead time, whereas lead time and backorder can be calculated precisely, which can provide exact cost of the system [33]. 


\subsection{Service level}

At present, service makes an important issue to avoid different unavoidable situation in the competitive market. Customers have been always attracted by the service facility of the company. Keeping in mind, service is considered here to increase the products popularity as well as market demand. Considering the maximum service level under stochastic environment Jodlbauer and Reitner [28] analyzed a multi-item production system for fixed safety stock. The service level directly effect on the optimal policy was shown by Bhat and Krishnamurthy [5]. A reverse logistic closed-loop supply chain always profitable for the supply chain players [16]. Nandra et al. [40] established that there was a relation between production rate and quality of the product. They proved that the rate of imperfection increases along with increase production rate. In the similar direction a multi-retailer sustainable supply chain for deteriorating items was proposed by Nandra et al. [41]. To obtain best service quality, it is necessary to invest some amount for preserving the deteriorate items [46,56]. Sepehri et al. [57] discussed about improvement in the quality of the production system, which enhance the service facilities of the industry. To attract the customers, Sarkar et al. [52] recently considered O2O system base production model with the service facility.

\subsection{Variable lead time}

In business sector, a valuable indicator through either online or offline mode is lead time. For the fully backorder case and long gap of lead time, popularity of the products gradually decreases. The lead time for the manufacturing system is directly dependent on order quantity. In an inventory model, Ben-Daya and Raouf [4] considered the concept of lead time without any shortage. For an inventory model, different strategies for the reduction of lead time under the stochastic demand were presented by Glock [18]. Through mixing the service with fluctuate lead time, Guchhait and Sarkar [21] proposed a review inventory system. As can be seen from the history of research, most of all research considered fixed and deterministic lead time but recently this idea is big helpless due to complex setups. Khan et al. [29] developed the effect of variable type of lead time for a supply chain. Through considering coordinating nature among the members, Heydari et al. [25] introduced a SCM with lead time under stochastic demand. A multi-national supply chain for promised delivery at early mentioned lead time was proposed by Noori-Daryan et al. [42]. The facility of lead time reduction through different ordering policies for reorder point was discussed by Li [33]. Recently, an advance SCM with lead time and its variance was proposed by Dey et al. [12]

\subsection{Variable ordering cost}

This is an essential investment among the necessary costs of the supply chain to make an order. Ordering cost reduction is the process used by the manufacturers to reduce their costs and increase their profits. It was shown from the previous research history that, Lee et al. [32] introduced an inventory system through applying some capital investment there was a significant reduction of ordering cost. They also focused on the lead time reduction strategies through the same investment of extra crashing cost. A stochastic inventory system was studid by Chao et al. [8] with fixed ordering cost. Through considering the trade credit financing approach Lou and Wang [35] considered an inventory system with considering ordering cost as decision variable. Bhuniya et al. [7] discussed the multi-retailer base supply chain, where ordering cost is variable due to different tariff plan. Recently, based on similar concept an O2O base SSCM was presented by Sarkar et al. [52]. Reworking is one of the best option to reduce the imperfect item of an imperfect manufacturing system, which directly helps to control the backorder situation [1].

\subsection{Backorder}

A backorder is an order for a good or service that cannot be fulfilled at the present time due to lack of supply. The item may not be on the manufacturer's available list but may still be in production, or the manufacturer may still need to produce more of the product. Srivastav and Agrawal [60] introduced a hybrid backorder inventory model where time-weighted backorder and fixed backorder is developed. A rationing problem base 
inventory system was examined by Bao et al. [3] under multiple demand classes. In this model, the authors developed a dynamic inventory system with backorder and for single item. An inventory system with multiple backorders under mixed lost sales was examined by ElHafsi et al. [15]. Recently, Mishra et al. [39] discussed an inventory system with backorder to inquire sustainable inventory management. Effect of fixed and linear backorder for the multi-item assembled product was calculated by Dey et al. [14]. In this model they considered a random rate for the defectiveness of the imperfect system.

With the help of backorder, several inventory, production, and SCM were illustrated in the literature. However, dual-channel SCM with the expected backorder through marginal value is still a gap in the literature. Moreover, calculation of lead time through variance and marginal value, where the demand of the product is calculated through the optimal value of the selling price and products were sold through online and offline channels, is still not studied yet. This study fills those research gaps.

\section{Problem explanation, symbols/memorandum, and Presumptions}

In this segment, the illustration of the model is presented, simultaneously the symbols with presumptions for the model are explained.

\subsection{Problem explanation}

A service upgraded O2O SCM is formulated in this current study. Due to the enormous applicability of the e-marketing strategy, recent research focuses on improving online retailing and offline marketing. The demand of both channels, i.e., online channel and offline channel, varies with the product's selling price. To upgrade the service's quality to the customer, controllable leadtime and variable variance are incorporated in this current study. By providing the knowledge of the exact waiting time, any industry can improve its service level. Thus, a marginal value of lead time and the marginal value of backorder are calculated in this study. Suppose the managers have exact knowledge of backorder, he can make a decision on safety stock, which enhances the system profit and improves the service level, which directly satisfies the customer.

Any online-to-offline system to place an order plays a major role, and this ordering cost can not permanently be fixed. Thus, by incorporating some investment, ordering cost can be diminish, which directly enhancing the system profit. During long-run, manufacturing system can be shiftted to an out-of-control state and start producing defective products. Those flawed or imperfect products can hamper the supply chain. To overcome this situation and provide better service to the customers, continuous investment is incorporated to upgrade the reliability of the production process.

Moreover, there will be different uncertainties that may arise to run a supply chain smoothly. A decentralize case also adopted to prove that trade-off between the players of the supply chain always provide better result.

\subsection{Notation}

Some notation and assumptions are adopted to illustrate the research, which are as follows:

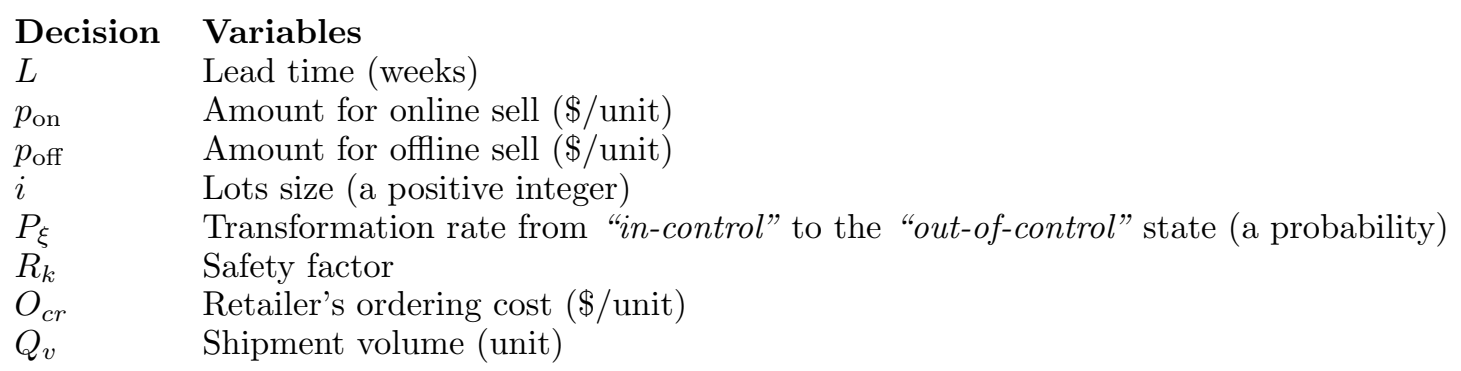




\section{Parameters}

$\begin{array}{ll}S_{c} & \text { Cost of replacing imperfect goods (\$/unit) } \\ S_{L} & \text { Lot size (units) } \\ D_{S} & \text { Demand rate of retailer, } D_{S}=A_{f} p_{\text {on }}{ }^{-\gamma}+\rho p_{\text {off }}{ }^{-\delta} \text { (units) } \\ R_{P} & \text { Manufacturer's rate of production, } R_{P}>D_{S} \text { (units/ time) } \\ C_{p m} & \text { Cost of unit purchase of retailer's }(\$ / \text { unit purchased) } \\ C_{T} & \text { Cycle time, } C_{T}=S_{L} / D_{S}=m Q_{v} / D_{S} \text { (time) } \\ F_{c c b} & \text { Cost related to fixed carbon emission cost }(\$ / \text { shipment) } \\ \xi_{0} & \text { Initially probability to the imperfect production rate (unit less) } \\ M_{h} & \text { Inventory holding cost for manufacturer }(\$ / \text { unit } / \text { unit time) } \\ C_{p h} & \text { Internet host purchasing cost for O2O installation }(\$ / \text { unit) } \\ \omega & \text { Portion of random defective production rate (follows certain distribution) } \\ S_{A} & \text { Setup cost of manufacturer }(\$) \\ \psi & \text { Standard normal probability density function } \\ \mu & \text { Mean lead time (time unit) } \\ A_{f} & \text { Fractional cost related to online sell ( } \$ / \text { investment) } \\ \sigma & \left.\text { (= } \frac{L}{\mu}\right) \text { the "coefficient of variance per time period" } \\ V_{c c b} & \text { Carbon emission cost related to retailer }(\$ / \text { unit) } \\ R_{h} & \text { Retailer holding cost }(\$ / \text { unit } / \text { unit time) }\end{array}$

\section{Parameters}

$\gamma \quad$ Parameter related to online price

$T_{F} \quad$ Fixed transportation cost $(\$ /$ shipment $)$

$T_{D} \quad$ Development cost of technology for O2O channeling ( $\$ /$ unit)

$\delta \quad$ Price elasticity parameter for offline price

$C_{u p} \quad$ Cost related to unit production of manufacturer (\$/unit)

$\rho \quad$ Parameter related to offline sell (\$/investment)

$\mu_{x} \quad$ Mean lead time demand

$O_{c r_{0}} \quad$ Cost related to initial order $(\$ /$ order $)$

$r_{k} \quad$ Reorder point for the retailer (unit)

A Fixed investment related to technology development $(\$ /$ cycle)

$C_{w d} \quad$ Cost for design web page for $\mathrm{O} 2 \mathrm{O}$ installation (\$/unit)

$\sigma_{L} \quad$ Variance of the lead time (unit less)

$C_{v w} \quad$ Cost related to web page visualization (\$/unit)

$\psi \quad$ Backorder cost (\$/unit)

$F_{c c v} \quad$ Manufacturer's fixed carbon emission cost (\$/shipment)

$V_{c c v} \quad$ Manufacturer's variable carbon emission cost $(\$ /$ unit)

$T_{V} \quad$ Cost related to variable transportation (\$/unit)

$\Phi \quad$ Cumulative distribution function

$E[B] \quad$ Number of quantity for expected backorder (unit)

$E[I] \quad$ Expected on-hand inventory

\subsection{Assumptions}

Following hypotheses are made to construct this O2O SCM:

(1) A savvy $\mathrm{O} 2 \mathrm{O}$ retailing is created for a particular item, where a particular producer and individual-retailer are the players of the SC.

(2) The perfect finished product be delivered to the retailer by offline channel, whereas, retailer use both channel for selling the product. Thus, to provide better service to the customer, the demands in both channels are based two different selling price. Thus, entire SCM demand is formulated as $D_{S}=A_{f} p_{\text {on }}{ }^{-\gamma}+\rho p_{\text {off }}{ }^{-\delta}[52]$. 
(3) Two investments are implied to upgrade the reliability of production process's and reduce ordering cost, which directly enhance the service [7].

(4) To give best service calculation of exact lead time and reduction in lead time two valuable features for any SCM. To obtain accurate LT, variance of the LT take a major role and which is obtain as $\sigma_{x}^{2}=L \sigma^{2}+\mu^{2} \sigma_{L}^{2}$ $[12]$.

(5) Due to imperfect manufacturing, backorder is arries, which is calculated through the marginal value and the inventory level depends on the distribution function of SF [33].

(6) A SSMD transportation policy is considered here under the O2O SCM. The producer applies multiple delivery technique after single slot production. Under the O2O environment, the SSMD arrangement makes the system more perfect way of business strategy. Due to transportation, constant and variable type of carbon outflow prices and transportation costs were thought to form the model for the revenue benefit as well as add up to the framework benefit [52].

\section{MAthematical MODEL}

The benefit of the entire SCM is overhauled through the O2O channeling system, which too makes a difference to optimize the entire framework benefit. In view of customer satisfaction some maintenance or servicing policies are adopted, where supply chain players earn revenue from those servicing or maintenance policy. The cost for supply chain players are as follows:

\subsection{Retailer's profit}

Retailer's inventory position is presented in the Figure 2.

Expected value of the inventory is calculated through weighted average of $E[I]$, which is distributed uniformly over $\left[r_{k}, r_{k}+Q_{v}\right]$ (see [12]). Therefore,

$$
\begin{aligned}
E[I] & =\frac{Q_{v}}{2}+\left(r_{k}-\mu_{x}\right)+E[B] \\
& =\frac{Q_{v}}{2}+R_{k} \sigma_{x}+\frac{\sigma_{x}^{2}}{Q_{v}}\left(\Phi^{2}\left(R_{k}\right)-\Phi^{2}\left(R_{k}+\frac{Q_{v}}{\sigma_{x}}\right)\right) .
\end{aligned}
$$

Since, safety stock $=$ reorder point, mean lead time demand $=r_{k}-\mu_{x}=R_{k} \sigma_{x}$.

Therefore, the costs related to retailer are presented as follows:

\subsubsection{Technology development cost}

This current study is created based on O2O SCM. To keep up with this O2O framework, smart technology is required. Hence, a few investments are necessary for advancement in technology along with few unit technology development costs. Thus, technology development cost for the SCM is given by

$$
(\mathrm{TDC})=A+\left(\frac{T_{D}\left(A_{f} p_{\mathrm{on}}^{-\gamma}+\rho p_{\mathrm{off}^{-\delta}}\right)}{Q_{v}}\right) .
$$

\subsubsection{Ordering cost with investment}

Placing order is one of the vital issue for O2O SCM. Nowadays, due to huge use of online platform to choose a particular product or to cancel an order, it is necessary that one has an internet connection in their smart phone or in any electronic device and for this connectivity, one has to pay certain amount to the service provider, which is also treated as ordering cost [52]. Since different provider has different tariff plan for internet connectivity, a retailer can invest certain amount to use this type of connectivity with less price. Thus, ordering cost for the whole supply chain is given as:

$$
\frac{O_{c r}\left(A_{f} p_{\mathrm{on}}^{-\gamma}+\rho p_{\mathrm{off}^{-\delta}}^{-\delta}\right)}{Q_{v}}+g \ln \frac{O_{c r_{0}}}{O_{c r}} .
$$




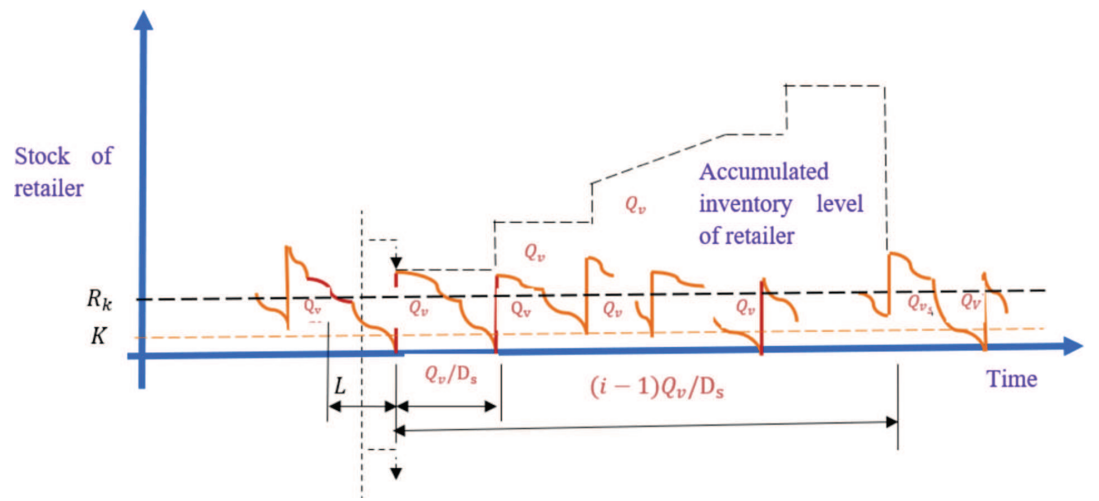

FIGURE 2. Inventory position of retailer.

\subsubsection{Holding cost}

After receiving the ordered quantity, retailer has to store them in some warehouse or he has to display those items in any retail shop. Thus, to keep those quantity, retailer needs some space and retailer have to pay certain amount to keep this product or to display this product in retail shop. This holding cost for the retailer is calculated through the marginal value of lead time and which is as follows (see [12]):

$$
R_{h}\left(\frac{Q_{v}}{2}+R_{k} \sigma_{x}+\frac{\sigma_{x}^{2}}{Q_{v}}\left(\Phi^{2}\left(R_{k}\right)-\Phi^{2}\left(R_{k}+\frac{Q_{v}}{\sigma_{x}}\right)\right)\right)+R_{h} \mu L
$$

\subsubsection{Expected backorder}

Since, expected inventory is calculated through weighted value of $E[I]$, similarly, expected backorder $E[B]$ calculated over $\left[r_{k}, r_{k}+Q_{v}\right]$ as [12]

$$
\begin{aligned}
E[B] & =\frac{1}{Q_{v}} \int_{r_{k}}^{r_{k}+Q_{v}} \sigma_{x} \Phi^{1}\left(\frac{x-L \mu}{\sigma_{x}}\right) \mathrm{d} x \\
& =\frac{\sigma_{x}^{2}}{Q_{v}}\left(\Phi^{2}\left(R_{k}\right)-\Phi^{2}\left(R_{k}+\frac{Q_{v}}{\sigma_{x}}\right)\right)
\end{aligned}
$$

where reorder point $r_{k}=L \mu+R_{k} \sigma_{x}$ and $\mu_{x}=L \mu$.

\subsubsection{Backorder cost}

Backorder cost incorporates costs incurred by a industry when it is incapable to instantly fill an order and guarantees the client that it will be completed with a afterward conveyance date.

In common, companies may include a few extra inventory measurements to get it and analyze the backorder cost in their supply chain [12]. This type of cost be such that

$$
\frac{\left(A_{f} p_{\mathrm{on}}{ }^{-\gamma}+\rho p_{\mathrm{off}}{ }^{-\delta}\right) \psi}{Q_{v}}\left[\frac{\sigma_{x}^{2}}{Q_{v}}\left(\Phi^{2}\left(R_{k}\right)-\Phi^{2}\left(R_{k}+\frac{Q_{v}}{\sigma_{x}}\right)\right)\right] .
$$

Therefore, entire cost component of retailer is given by

$$
\begin{aligned}
\mathrm{TC}_{r}\left(Q_{v}, p_{\text {on }}, p_{\text {off }}, L, O_{c r}, R_{k}\right) & =\mathrm{HC}+\mathrm{OC}+(\mathrm{TDC})+\mathrm{BC}+\mathrm{LTCC} \\
& =\left[R_{h}\left(\frac{Q_{v}}{2}+R_{k} \sigma_{x}+\frac{\sigma_{x}^{2}}{Q_{v}}\left(\Phi^{2}\left(R_{k}\right)-\Phi^{2}\left(R_{k}+\frac{Q_{v}}{\sigma_{x}}\right)\right)\right)+R_{h} \mu L\right]
\end{aligned}
$$




$$
\begin{aligned}
& +\frac{O_{c r}\left(A_{f} p_{\mathrm{on}}{ }^{-\gamma}+\rho p_{\mathrm{off}}{ }^{-\delta}\right)}{Q_{v}}+g \ln \frac{O_{c r_{0}}}{O_{c r}}+A+\frac{T_{D}\left(A_{f} p_{\mathrm{on}}{ }^{-\gamma}+\rho p_{\mathrm{off}}{ }^{-\delta}\right)}{Q_{v}} \\
& +\frac{\left(A_{f} p_{\mathrm{on}}^{-\gamma}+\rho p_{\mathrm{off}^{-}}{ }^{-\delta}\right) \psi}{Q_{v}}\left[\frac{\sigma_{x}^{2}}{Q_{v}}\left(\Phi^{2}\left(R_{k}\right)-\Phi^{2}\left(R_{k}+\frac{Q_{v}}{\sigma_{x}}\right)\right)\right] .
\end{aligned}
$$

Now, the marginal values respect to lead time is calculated as

$$
\begin{aligned}
\mathrm{MVL}= & \frac{\partial \mathrm{TC}_{B}}{\partial L}=R_{h} \mu+R_{h} R_{k} \frac{\partial \sigma_{x}}{\partial L} \\
& +\frac{Q_{v} R_{h}+\left(A_{f} p_{\mathrm{on}^{-\gamma}}{ }^{-\gamma} \rho p_{\mathrm{off}^{-}}{ }^{-\delta}\right) \psi}{Q_{v}^{2}} \frac{\partial}{\partial L}\left[\sigma_{x}^{2}\left(\Phi^{2}\left(R_{k}\right)-\Phi^{2}\left(R_{k}+\frac{Q_{v}}{\sigma_{x}}\right)\right)\right] .
\end{aligned}
$$

Similarly, marginal values respect to $\sigma^{2}$ (variance of lead time duration) obtain as

$$
\frac{\partial \mathrm{TC}_{r}}{\partial \sigma_{L}^{2}}=R_{h} R_{k} \frac{\partial \sigma_{x}}{\partial \sigma_{L}^{2}}+\frac{Q_{v} R_{h}+\left(A_{f} p_{\mathrm{on}^{-\gamma}}+\rho p_{\mathrm{off}^{-}}\right) \psi}{Q_{v}^{2}} \frac{\partial}{\partial \sigma_{L}^{2}}\left(\sigma_{x}^{2}\left(\Phi^{2}\left(R_{k}\right)-\Phi^{2}\left(R_{k}+\frac{Q_{v}}{\sigma_{x}}\right)\right)\right) .
$$

Putting $\sigma_{x}^{2}=L \sigma^{2}+\mu^{2} \sigma_{L}^{2}$

$$
\begin{aligned}
\mathrm{MVL}= & R_{h} \mu+R_{h} R_{k} \frac{\sigma^{2}}{2 \sqrt{L \sigma^{2}+\mu^{2} \sigma_{L}^{2}}}+\frac{Q_{v} H_{r}+\left(A_{f} p_{\mathrm{on}^{-\gamma}}+\rho p_{\mathrm{off}^{-\delta}}\right) \psi}{Q_{v}^{2}} \\
& \times \frac{\sigma^{2}}{2 \sqrt{L \sigma^{2}+\mu^{2} \sigma_{L}^{2}}}\left(\sqrt{L \sigma^{2}+\mu^{2} \sigma_{L}^{2}}\left(\Phi^{2}\left(R_{k}\right)-\Phi^{2}\left(R_{k}+\frac{Q_{v}}{\sigma_{x}}\right)\right)-Q_{v} \Phi\left(R_{k}+\frac{Q_{v}}{\sigma_{x}}\right)\right) .
\end{aligned}
$$

The revenue for the retailer is $=\left(p_{\text {on }}-C_{p m}\right) A_{f} p_{\text {on }}^{-\gamma}+\left(p_{\text {off }}-C_{p m}\right) \rho p_{\text {off }}^{-\delta}$.

Now, the retailer's profit can be calculated as

$$
\begin{aligned}
\mathrm{TP}_{r}\left(Q_{v}, R_{k}, L, p_{\text {on }}, p_{\text {off }}, O_{c r}\right) & =\left(\left(p_{\text {on }}-C_{p m}\right) A_{f} p_{\text {on }}^{-\gamma}+\left(p_{\text {off }}-C_{p m}\right) \rho p_{\text {off }}^{-\delta}\right)-\operatorname{TC}_{B}\left(Q_{v}, p_{\text {on }}, p_{\text {off }}, \sigma, T_{l}, O_{c r}\right) \\
& =\left(\left(p_{\text {on }}-C_{p m}\right) A_{f} p_{\text {on }}^{-\gamma}+\left(p_{\text {off }}-C_{p m}\right) \rho p_{\text {off }}^{-\delta}\right) \\
& -\left[\left[R_{h}\left(\frac{Q_{v}}{2}+R_{k} \sigma_{x}+\frac{\sigma_{x}^{2}}{Q_{v}}\left(\Phi^{2}\left(R_{k}\right)-\Phi^{2}\left(R_{k}+\frac{Q_{v}}{\sigma_{x}}\right)\right)\right)+R_{h} \mu L\right]\right. \\
& +\frac{O_{c r}\left(A_{f} p_{\text {on }}-\gamma+\rho p_{\text {off }}-\delta\right.}{Q_{v}}+g \ln \frac{O_{c r_{0}}}{O_{c r}}+A+\frac{T_{D}\left(A_{f} p_{\text {on }}-\gamma+\rho p_{\text {off }}-\delta\right)}{Q_{v}} \\
& +\frac{\left(A_{f} p_{\text {on }}^{-\gamma}+\rho p_{\text {off }}^{-\delta}\right) \psi}{Q_{v}}\left[\frac{\sigma_{x}^{2}}{Q_{v}}\left(\Phi^{2}\left(R_{k}\right)-\Phi^{2}\left(K+\frac{Q}{\sigma_{x}}\right)\right)\right] .
\end{aligned}
$$

\subsection{Profit of the manufacturer}

Figure 3 represent the position of manufacturer's stock. The costs related to manufacturer are presented below.

\subsubsection{Cost related to setup for O2O SCM}

Setup cost is related with the setting up of apparatus before beginning manufacturing. Setup cost is one of the crucial costs of any SC, setup cost for this O2O SCM is calculated as follows.

$$
\frac{S_{A}\left(A_{f} p_{\mathrm{on}}^{-\gamma}+\rho p_{\mathrm{off}^{-}}{ }^{-\delta}\right)}{i Q_{v}} .
$$




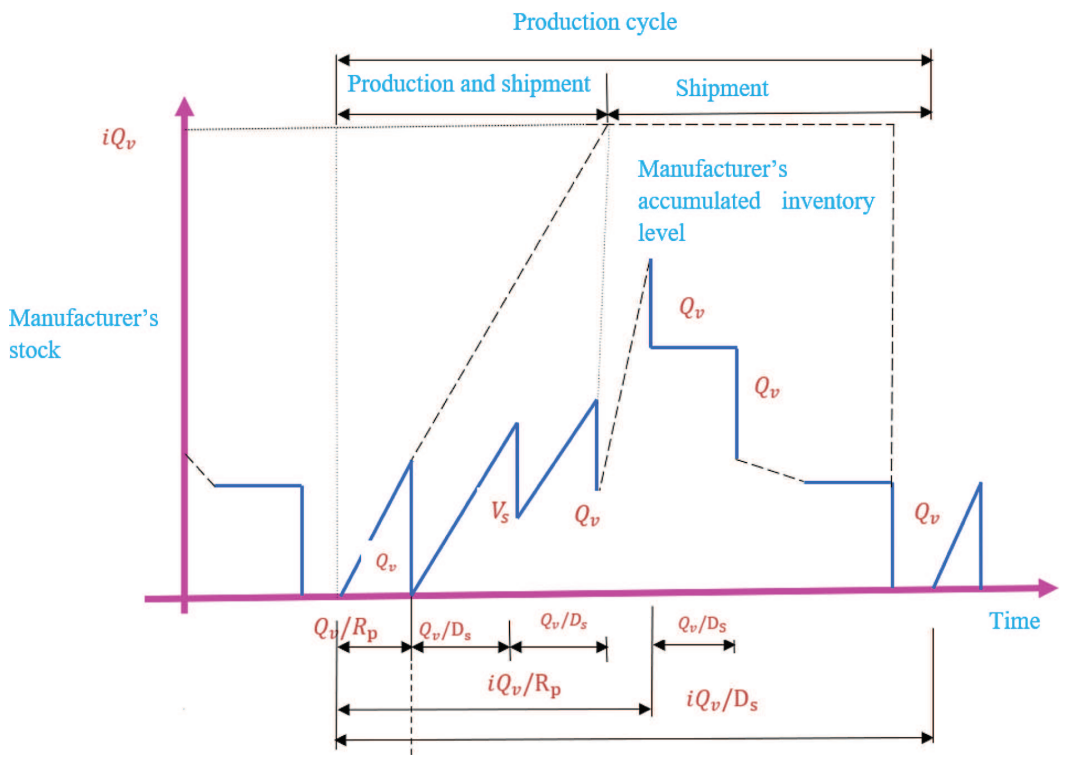

FIGURE 3. Inventory position of the manufacturer.

\subsubsection{Cost related to transportation for O2O SCM}

Transporting the raw materials and manufactured products is too much important for SC. The management may consider several transportation strategies to diminish the transportation cost of the SC. Many manufacturer would recognize transportation supply chain permeability as an essential objective within the company. The SSMD transportation strategy is utilized in this study to transport products from manufacturer's warehouse to retailer warehouse. Both fixed and variable transportation cost for the manufacturer is considered in this study.

$$
T_{F} i+T_{V}\left(A_{f} p_{\text {on }}^{-\gamma}+\rho p_{\text {off }}^{-\delta}\right) .
$$

\subsubsection{Cost for holding the product}

Cost related to hold the product for manufacturer is the foremost measurable cost and can be translated as the most or as it was taken a toll on a stock without any respect for the different costs such as shortage and ordering costs.

Following equation present the average inventory for the manufacturer

$$
=\left[\frac{Q_{v}}{2}+\frac{(i-2) Q_{v}}{2}\left(1-\frac{\left(A_{f} p_{\mathrm{on}}^{-\gamma}+\rho p_{\text {off }}{ }^{-\delta}\right)}{(1-\omega) R_{P}}\right)\right] .
$$

Therefore, total cost for hold the entire product in manufacturer's warehouse is provided as (see [58])

$$
=M_{h}\left[\frac{Q_{v}}{2}+\frac{(i-2) Q_{v}}{2}\left(1-\frac{\left(A_{f} p_{\mathrm{on}}^{-\gamma}+\rho p_{\text {off }}{ }^{-\delta}\right)}{(1-\omega) R_{P}}\right)\right] .
$$

\subsubsection{Cost related to carbon emission for O2O SCM}

A carbon cost gives a financial flag in contaminating businesses to diminish and in the long run, suspend their harmful exercises emitting carbon dioxide and other green house gas emissions. A carbon cost strategy 
broadly concurred to be the foremost efficient way for countries to reduce global warming. Due to using the concept of SSMD transportation strategy, the cost related to carbon emission is implied here. Entire cost for carbon emission is calculated by summing fixed and variable carbon emission, which is given by

$$
\mathrm{CE}_{v}=\left(F_{c c v}\right) i+V_{c c v}\left(A_{f} p_{\mathrm{on}}{ }^{-\gamma}+\rho p_{\text {off }}{ }^{-\delta}\right) .
$$

\subsubsection{Capital investment to improve the reliability of the manufacturing process}

When a manufacturing system run over a long time, the reliability of the process may be reduced for different issues like machinery problem, unskilled labour and many more. The production process may start to produce faulty product in "out-of-control" situation. The amount of faulty items are $\frac{S_{c}\left(A_{f} p_{\mathrm{or}}{ }^{-\gamma}+\rho p_{\mathrm{off}}-\delta\right) S_{L} P_{\xi}}{2}$. Some "investment is required to improve the reliability and the quality of the production process", which directly enhance the service quality. Thus, the investment for improvement of the production process is given by

$$
b \ln \left(\frac{\xi_{0}}{P_{\xi}}\right)
$$

\subsubsection{Cost related to O2O installation}

Some costs are required to implementing intelligent $\mathrm{O} 2 \mathrm{O}$ service, which enhance the service of the manufacturer. For implementing the $\mathrm{O} 2 \mathrm{O}$ technology, costs are required for purchasing the domain, designing the web page, labour, and visualization for web page (see [52]). Thus, cost related to $\mathrm{O} 2 \mathrm{O}$ installation is provided by

$$
\mathrm{O}_{2} \mathrm{O}_{I C}=C_{w d}+C_{p h}+C_{v w}+C_{l w}
$$

Therefore, total cost related to $\mathrm{O} 2 \mathrm{O}$ technology installation is provided as

$$
\frac{\left(C_{w d}+C_{p h}+C_{v w}+C_{l w}\right)\left(A_{f} p_{\mathrm{on}}^{-\gamma}+\rho p_{\text {off }}^{-\delta}\right)}{Q_{v} i} .
$$

Thus, entire cost for the manufacturer with O2O SCM under improved service quality is given as

$$
\begin{aligned}
& \mathrm{TC}_{m}\left(Q_{v}, p_{\mathrm{on}}, p_{\text {off }}, P_{\xi}, i\right)=\frac{\left(A_{f} p_{\mathrm{on}}{ }^{-\gamma}+\rho p_{\mathrm{off}}{ }^{-\delta}\right)}{i Q_{v}}\left(C_{w d}+C_{p h}+C_{v w}+C_{l w}\right)+\frac{S_{A}\left(A_{f} p_{\mathrm{on}}{ }^{-\gamma}+\rho p_{\mathrm{off}}{ }^{-\delta}\right)}{i Q_{v}} \\
& +M_{h}\left[\frac{Q_{v}}{2}+\frac{(m-2) Q_{v}}{2}\left(1-\frac{\left(A_{f} p_{\mathrm{on}^{-\gamma}}+\rho p_{\mathrm{off}^{-\delta}}\right)}{(1-\omega) R_{P}}\right)\right]+b \ln \frac{P_{\theta_{0}}}{P_{\xi}} \\
& +\frac{i S_{c}\left(A_{f} p_{\mathrm{on}}{ }^{-\gamma}+\rho p_{\mathrm{off}}{ }^{-\delta}\right) Q_{v} P_{\theta}}{2}+\frac{\left(T_{F}+F_{c c v}\right)\left(A_{f} p_{\mathrm{on}}{ }^{-\gamma}+\rho p_{\mathrm{off}}{ }^{-\delta}\right)}{Q_{v}} \\
& +\left(T_{V}+V_{c c v}\right)\left(A_{f} p_{\text {on }}^{-\gamma}+\rho p_{\text {off }}^{-\delta}\right) .
\end{aligned}
$$

Now, revenue for the manufacturer is calculated as $\left(C_{p m}-C_{u p}\right) A_{f} p_{\text {on }}^{-\gamma}+\left(C_{p m}-C_{u p}\right) \rho p_{\text {off }}^{-\delta}$.

Therefore, profit for the manufacturer presented as

$$
\begin{aligned}
& \mathrm{TP}_{m}\left(Q_{v}, i, p_{\text {on }}, p_{\text {off }}, P_{\xi}\right)=\left(\left(C_{p m}-C_{u p}\right)\left(A_{f} p_{\text {on }}^{-\gamma}+\rho p_{\text {off }}^{-\delta}\right)\left(A_{f} p_{\text {on }}^{-\gamma}+\rho p_{\text {off }}^{-\delta}\right)\right)-\mathrm{TC}_{m}\left(Q_{v}, R_{f}, s_{p}, P_{\theta}, q_{v}, s\right) \\
& =\left(\left(C_{p m}-C_{u p}\right)\left(A_{f} p_{\text {on }}^{-\gamma}+\rho p_{\text {off }}^{-\delta}\right)\left(A_{f} p_{\text {on }}^{-\gamma}+\rho p_{\text {off }}^{-\delta}\right)\right) \\
& -\left[\frac{\left(A_{f} p_{\mathrm{on}}{ }^{-\gamma}+\rho p_{\mathrm{off}}{ }^{-\delta}\right)}{i Q_{v}}\left(C_{w d}+C_{p h}+C_{v w}+C_{l w}\right)+\frac{S_{A}\left(A_{f} p_{\mathrm{on}}{ }^{-\gamma}+\rho p_{\mathrm{off}}{ }^{-\delta}\right)}{i Q_{v}}\right. \\
& +b \ln \frac{\xi_{0}}{P_{\xi}}+\frac{i S_{c}\left(A_{f} p_{\mathrm{on}^{-\gamma}}+\rho p_{\mathrm{off}^{-}}{ }^{-\delta}\right) Q_{v} P_{\xi}}{2}+M_{h}\left[\frac{Q_{v}}{2}+\frac{(i-2) Q_{v}}{2}\right.
\end{aligned}
$$




$$
\begin{aligned}
& \left.\times\left(1-\frac{\left(A_{f} p_{\text {on }}{ }^{-\gamma}+\rho p_{\text {off }}^{-\delta}\right)}{(1-\omega) R_{P}}\right)\right]+\frac{\left(T_{F}+F_{c c v}\right)\left(A_{f} p_{\text {on }}{ }^{-\gamma}+\rho p_{\text {off }}{ }^{-\delta}\right)}{Q_{v}} \\
& \left.+\left(V_{c c v}+T_{V}\right)\left(A_{f} p_{\text {on }}{ }^{-\gamma}+\rho p_{\text {off }}{ }^{-\delta}\right)\right] .
\end{aligned}
$$

\section{Solution technique}

To prove that the coordination between the players is more beneficial, in this section two cases are discussed. First, we optimize the value for each player naming decentralize case and then we proposed the methodology for centralize case, where joint profit is optimized based on the value of decision variables

\subsection{Decentralize case}

To obtain the value of the decision variables, one can take the first order partial derivative of Equation 4.2 and equating to zero the optimal values are obtain as follows

$$
\begin{aligned}
& p_{o n_{r}}^{*}=\frac{\gamma}{Q_{v}(1-\gamma)}\left(O_{c r}+T_{D}+\frac{\psi \sigma_{x}^{2}}{Q_{v}}\left(\Phi^{2}\left(R_{k}\right)-\Phi^{2}\left(K+\frac{Q}{\sigma_{x}}\right)\right)-C_{p m}\right) \\
& p_{o f f_{r}}^{*}=\frac{\delta}{Q_{v}(1-\delta)}\left(O_{c r}+T_{D}+\frac{\psi \sigma_{x}^{2}}{Q_{v}}\left(\Phi^{2}\left(R_{k}\right)-\Phi^{2}\left(K+\frac{Q}{\sigma_{x}}\right)\right)-C_{p m}\right)
\end{aligned}
$$

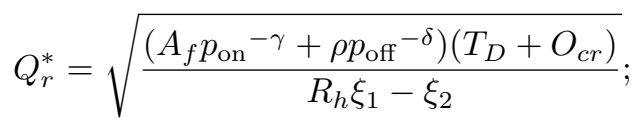

where, $\xi_{1}=\left[\frac{1}{2}-\frac{\sigma_{x}^{2}}{Q_{v}^{2}}\left(\Phi^{2}\left(R_{k}\right)-\Phi^{2}\left(R_{k}+\frac{Q_{v}}{\sigma_{x}}\right)\right)-\frac{\sigma_{x}}{Q_{v}} \Phi^{1}\left(R_{k}+\frac{Q_{v}}{\sigma_{x}}\right)\right]$

$$
\begin{aligned}
\xi_{2}= & \frac{2\left(A_{f} p_{\mathrm{on}}{ }^{-\gamma}+\rho p_{\mathrm{off}^{-\delta}}\right) \sigma_{x}^{2} \psi}{3 Q^{3}}\left(\Phi^{2}\left(R_{k}\right)-\Phi^{2}\left(R_{k}+\frac{Q_{v}}{\sigma_{x}}\right)\right) \\
& +\frac{\left(A_{f} p_{\mathrm{on}}{ }^{-\gamma}+\rho p_{\mathrm{off}}{ }^{-\delta}\right) \sigma_{x} \psi}{Q_{v}} \Phi^{\prime}\left(R_{k}+\frac{Q_{v}}{\sigma_{x}}\right) \\
O_{c r}^{*}= & \frac{g Q_{v}}{\left(A_{f} p_{\mathrm{on}}{ }^{-\gamma}+\rho p_{\mathrm{off}}{ }^{-\delta}\right)} \\
L^{*}= & \frac{1}{\sigma^{2}}\left[\mu^{2} \sigma_{L}^{2}-\frac{\sigma^{4} K^{2} R_{h}^{2}}{4\left(\mu R_{h}+\xi_{3}\right)^{2}}\right],
\end{aligned}
$$$$
+\frac{\left(A_{f} p_{\mathrm{on}}{ }^{-\gamma}+\rho p_{\mathrm{off}}{ }^{-\delta}\right) \sigma_{x} \psi}{Q_{v}} \Phi^{\prime}\left(R_{k}+\frac{Q_{v}}{\sigma_{x}}\right)
$$

where, $\xi_{3}=\left(R_{h}+\frac{\left(A_{f} p_{\mathrm{on}}^{-\gamma}+\rho p_{\mathrm{off}^{-\delta}}\right)}{Q_{v}}\right)\left[\left(\Phi^{2}\left(R_{k}\right)-\Phi^{2}\left(R_{k}+\frac{Q_{v}}{\sigma_{x}}\right)\right) \frac{2 \sigma_{x}}{Q_{v}} \frac{\partial \sigma_{x}}{\partial L}\right.$

$$
\left.+\Phi^{1}\left(R_{k}+\frac{Q_{v}}{\sigma_{x}}\right) \frac{\partial \sigma_{x}}{\partial L}\right]
$$

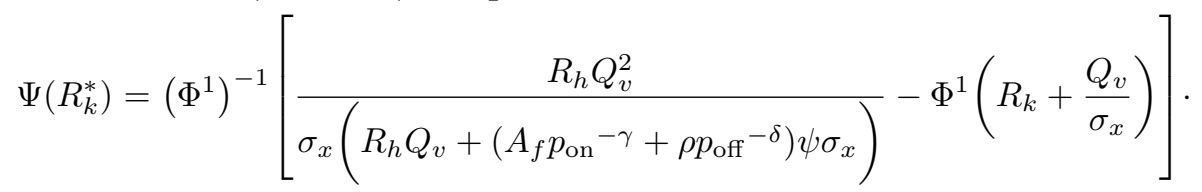

The detail calculation are provided in Appendices A and B. 
To prove the sufficient condition of the concavity, one have to calculate the Hessian matrix and all principal minor of the Hessian matrix must be negative definite or negative semi-definite.

To prove the concavity of the Equation 4.2 with respect to decision variables, please see Appendices A-G.

Now, optimum result for the manufacturer are calculated as follows

$$
\begin{aligned}
& Q_{v_{p}}^{*}=\sqrt{\frac{\left(A_{f} p_{\mathrm{on}}{ }^{-\gamma}+\rho p_{\mathrm{off}}{ }^{-\delta}\right)\left[F_{c c v}+T_{F}+\frac{\left(C_{w d}+C_{p h}+C_{v w}+C_{l w}+S_{A}\right)}{m}\right]}{i P_{\xi} S_{c}\left(A_{f} p_{\mathrm{on}}{ }^{-\gamma}+\rho p_{\mathrm{off}}{ }^{-\delta}\right)+M_{h}\left(1+(i-2)\left(1-\frac{\left(A_{f} p_{\mathrm{on}}-\gamma+\rho p_{\mathrm{off}}-\delta\right)}{(1-\omega) R_{P}}\right)\right)}} \\
& P_{\xi}=\frac{2 b}{i Q_{v} S_{c}\left(A_{f} p_{\mathrm{on}^{-\gamma}}{ }^{-\gamma} \rho p_{\mathrm{off}^{-\delta}}\right)} .
\end{aligned}
$$

Now to check the concavity of the profit function of the manufacturer, one can take the second order partial derivative with respect to $Q$, and $P_{\xi}$. The second order partial derivatives are as follows:

$$
\begin{aligned}
\frac{\partial^{2} T P_{m}}{\partial Q^{2}} & =-\frac{2\left(A_{f} p_{\text {on }}{ }^{-\gamma}+\rho p_{\text {off }}^{-\delta}\right)}{i Q_{v}^{3}}\left[i F_{c c v}+i T_{F}+C_{w d}+C_{p h}+C_{v w}+C_{l w}+S_{A}\right]<0 \\
\frac{\partial^{2} T P_{m}}{\partial P_{\xi}^{2}} & =-\frac{b}{P_{\xi}^{2}}<0 .
\end{aligned}
$$

Above equations (5.9) and (5.10) prove the necessary condition for concavity.

Now, to fulfil the sufficient condition of the concavity one have to calculate the value of the principal minor of the Hessian matrix. The calculation for the Hessian matrix as follows:

$$
H_{22}=\left|\begin{array}{cc}
\frac{\partial^{2} T P_{m}(\cdot)}{\partial Q_{v}^{2}} & \frac{\partial^{2} T P_{m}(\cdot)}{\partial Q_{v} \partial P_{\xi}} \\
\frac{\partial^{2} T P_{m}}{\partial P_{\xi} \partial Q_{v}} & \frac{\partial^{2} T P_{m}(\cdot)}{\partial P_{\xi}^{2}}
\end{array}\right|=\frac{\partial^{2} \chi(\cdot)}{\partial A_{V}^{2}} \frac{\partial^{2} \chi(\cdot)}{\partial \theta^{2}}=\frac{\partial^{2} T P_{m}(\cdot)}{\partial Q_{v}^{2}} \frac{\partial^{2} T P_{m}(\cdot)}{\partial P_{\xi}^{2}}-\left[\frac{-i S_{c}\left(A_{f} p_{\mathrm{on}}{ }^{-\gamma}+\rho p_{\text {off }}{ }^{-\delta}\right)}{2}\right]^{2} .
$$

By using the value of the equations (5.9) and (5.10) in the equation (5.11), one can state that the value of the equation (5.11) obviously grater then zero. Now, one can say that the profit for the manufacturer is globally optimal by using the conditions, which are obtain from equations (5.9)-(5.11).

\subsection{Centralized case}

Now the total profit of the retailer's and manufacturer's are presented by equations (4.2) and (4.4) respectively. Therefore, expected joint profit for entire O2O SCM is calculated as

$$
\begin{aligned}
& \text { Total profit }\left(Q_{v}, i, L, p_{\text {on }}, p_{\text {off }}, P_{\xi}, O_{c r}, R_{k}\right) \\
& =\left(\left(p_{\text {on }}-C_{u p}\right) A_{f} p_{\text {on }}^{-\gamma}+\left(p_{\text {off }}-C_{u p}\right) \rho p_{\text {off }}^{-\delta}\right)-\mathrm{TC}\left(Q_{v}, i, L, p_{\text {on }}, p_{\text {off }}, P_{\xi}, O_{c r}, R_{k}\right) \\
& =\left(\left(p_{\text {on }}-C_{\text {up }}\right) A_{f} p_{\text {on }}^{-\gamma}+\left(p_{\text {off }}-C_{\text {up }}\right) \rho p_{\text {off }}^{-\delta}\right) \\
& -\left[\left[R_{h}\left(\frac{Q_{v}}{2}+R_{k} \sigma_{x}+\frac{\sigma_{x}^{2}}{Q_{v}}\left(\Phi^{2}\left(R_{k}\right)-\Phi^{2}\left(R_{k}+\frac{Q_{v}}{\sigma_{x}}\right)\right)\right)+R_{h} \mu L\right]\right. \\
& +\frac{O_{c r}\left(A_{f} p_{\mathrm{on}}{ }^{-\gamma}+\rho p_{\mathrm{off}}{ }^{-\delta}\right)}{Q_{v}}+g \ln \frac{O_{c r_{0}}}{O_{c r}}+A+\frac{T_{D}\left(A_{f} p_{\mathrm{on}^{-\gamma}}{ }^{-\gamma}+\rho p_{\mathrm{off}}{ }^{-\delta}\right)}{Q_{v}} \\
& +\frac{\left(A_{f} p_{\text {on }}^{-\gamma}+\rho p_{\text {off }}^{-\delta}\right) \psi}{Q_{v}}\left[\frac{\sigma_{x}^{2}}{Q_{v}}\left(\Phi^{2}\left(R_{k}\right)-\Phi^{2}\left(R_{k}+\frac{Q_{v}}{\sigma_{x}}\right)\right)\right] \\
& +\frac{\left(A_{f} p_{\mathrm{on}}^{-\gamma}+\rho p_{\mathrm{off}}^{-\delta}\right)}{i Q_{v}}\left(C_{w d}+C_{p h}+C_{v w}+C_{l w}+S_{A}\right)
\end{aligned}
$$




$$
\begin{aligned}
& +M_{h}\left[\frac{Q_{v}}{2}+\frac{(i-2) Q_{v}}{2}\left(1-\frac{\left(A_{f} p_{\mathrm{on}}{ }^{-\gamma}+\rho p_{\mathrm{off}}{ }^{-\delta}\right)}{(1-\omega) R_{P}}\right)\right]+b \ln \frac{\xi_{0}}{P_{\xi}} \\
& +\frac{i S_{c}\left(A_{f} p_{\mathrm{on}}{ }^{-\gamma}+\rho p_{\mathrm{off}}{ }^{-\delta}\right) Q_{v} P_{\xi}}{2}+\frac{\left(T_{F}+F_{c c v}\right)\left(A_{f} p_{\mathrm{on}}{ }^{-\gamma}+\rho p_{\mathrm{off}^{-\delta}}\right)}{Q_{v}} \\
& \left.+\left(T_{V}+V_{c c v}\right)\left(A_{f} p_{\text {on }}{ }^{-\gamma}+\rho p_{\text {off }}{ }^{-\delta}\right)\right] .
\end{aligned}
$$

Now, for a fixed value of the number of shipments that is for fixed $i$, the value of the other decision variables for the centralized case are obtained as follows:

$$
\begin{aligned}
& p_{\mathrm{on}}^{*}=\frac{\gamma \Pi_{1}}{\gamma-1} \\
& p_{\text {off }}^{*}=\frac{\delta \Pi_{1}}{\delta-1} \\
& O_{c r}^{*}=\frac{g Q_{v}}{\left(A_{f} p_{\mathrm{on}^{-\gamma}}{ }^{-\gamma} \rho p_{\mathrm{off}^{-\delta}}\right)}
\end{aligned}
$$

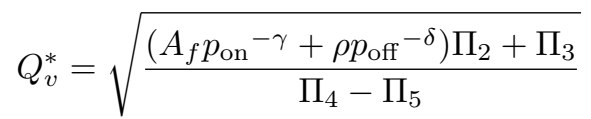

$$
\begin{aligned}
& P_{\xi}^{*}=\frac{2 b}{i Q_{v} S_{c}\left(A_{f} p_{\text {on }}{ }^{-\gamma}+\rho p_{\text {off }^{-\delta}}\right)} \\
& L^{*}=\frac{1}{\sigma^{2}}\left[\mu^{2} \sigma_{L}^{2}-\frac{\sigma^{4} R_{K}^{2} R_{h}^{2}}{4\left(\mu R_{h}+\psi_{3}\right)^{2}}\right],
\end{aligned}
$$

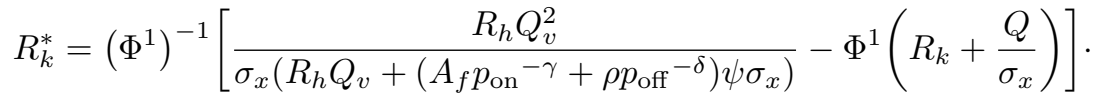

The values of the first order and second order partial derivatives and all others values are provided in the Appendices A-C.

To prove the sufficient condition for the concavity, one can utilize the following propositions.

Proposition 5.1. All first order principal minors of the Hessian matrix are less then zero due to the positive value of $b$, and $P_{\xi}$.

Proof. Please see Appendices C and D.

Proposition 5.2. All second order principal minors of the Hessian matrix are grater then zero due to the positive value of $b, g, O_{c r}$ and $P_{\xi}$.

Proof. Please see Appendices C and D.

Proposition 5.3. All third order principal minors of the Hessian matrix are less then zero due to the positive value of $\Gamma_{2} b, g, O_{c r}$ and $P_{\xi}$.

Proof. Please see Appendices C and D.

Proposition 5.4. All fourth order principal minors of the Hessian matrix are grater then zero if $\Gamma_{2}>\Theta_{9}$, and $\Gamma_{3}>\Theta_{9}$. Since, one can know that $\Gamma_{2} \Gamma_{3}>\Theta_{9}^{2}$, if and only if $\Gamma_{2}>\Theta_{9}$, and $\Gamma_{3}>\Theta_{9}$.

Proof. Please see Appendices C and E. 
Proposition 5.5. All fifth order principal minors of the Hessian matrix are less then zero if $\frac{b g \Gamma_{4}}{P_{\xi}^{2} O_{c r}^{2}}>$ $\frac{g}{O_{c r}^{2}}\left(\frac{A_{f} i Q_{v} S_{c} \gamma p_{\mathrm{on}}^{-1-\gamma}}{2}\right)^{2}$ and follows Proposition 5.4.

Proof. Please see Appendices C and F.

Proposition 5.6. All sixth order principal minors of the Hessian matrix are grater then zero if summation of first and third term of the right hand side of the Appendix $G$ is grater then the second term of the right hand side and follows Proposition 5.5.

Proof. Please see Appendices C and G.

Proposition 5.7. All seventh order principal minors of the Hessian matrix are less then zero if summation of first and third and last term of the right hand side of the Appendix $H$ is grater then the second term of the right hand side and follows Proposition 5.6.

Proof. Please see Appendices C and H.

From the above propositions one can conclude that the values of the leading principal minors are alternate in sign. It is well known that if leading principal minors of the Hessian matrix are alternate in sign, then the function is concave under the optimum value of the decision maker variables. Finally, the sufficient conditions for the concavity is approved by above discussion.

\section{NumericAl EXPERIMENT}

\subsection{Optimal result for decentralize case}

The value of the parametric for the decentralize case are obtain from Sarkar et al. [52] as follows: $R_{h}=$ $\$ 1.6 /$ unit, $\mu=2, O_{c_{0}}=\$ 450, g=60, A=\$ 60, \psi=\$ 5 /$ unit, $S_{A}=\$ 3 /$ unit, $C_{w d}=\$ 0.5 /$ unit, $C_{p h}=$ $\$ 2 /$ unit, $C_{v w}=\$ 10 /$ unit, $T_{F}=\$ 2 /$ unit, $C_{l w}=\$ 2 /$ unit, $R_{P}=150$ unit, $T_{V}=\$ 0.5 /$ unit $P_{c v}=\$ 2.6 /$ unit, $M_{h}=\$ 2.6 /$ unit, $b=0.01, T_{D}=\$ 2 /$ unit, $F_{c c v}=\$ 3 /$ unit, $V_{c c v}=\$ 8 /$ unit, $P_{\theta_{0}}=0.0001$. The value related to Uniform distribution are $(0.03,0.07)$; related to Triangular distribution are $(0.04,0.07,0.05)$; and related to Beta distribution are $(0.03,0.07)$. Only the changed values are $A_{f}=2000, \rho=2000, \gamma=1.3, \delta=1.5$, $C_{p m}=\$ 10 /$ unit, then the optimal result for retailer and manufacturer for decentralize case are provided in Tables 2 and 3 respectively.

Now, the total system profit for the decentralize case, when defective rate follows uniform distribution is given by $\$ 1741.99$ per cycle. Similarly, for a triangular distributed defective rate provide the profit $\$ 1741.80$ per cycle. Again, if defective rate follows double triangular distribution, then the system profit is given by $\$ 1741.54$ per cycle. Finally, if the defective rate follows a beta distribution then the profit is $\$ 1765.36$ per cycle.

TABLE 2. Optimum values for retailer.

\begin{tabular}{lllllll}
\hline \hline $\begin{array}{l}Q_{v}^{*} \\
\text { (units) }\end{array}$ & $\begin{array}{l}p_{\text {on }}^{*} \\
(\$ / \text { unit })\end{array}$ & $\begin{array}{l}p_{\text {off }}^{*} \\
(\$ / \text { unit })\end{array}$ & $\begin{array}{l}R_{k} \\
\text { (unit) }\end{array}$ & $\begin{array}{l}L^{*} \\
\text { (week) }\end{array}$ & $\begin{array}{l}O_{c r}^{*} \\
(\$ / \text { cycle })\end{array}$ & $\begin{array}{l}T P_{r} \\
(\$ / \text { cycle) }\end{array}$ \\
\hline 83.18 & 40.43 & 58.39 & 8 & 3 & 278.97 & 487.42 \\
\hline
\end{tabular}


TABLE 3. Manufacturer's optimum values.

\begin{tabular}{lllll}
\hline \hline & $\begin{array}{l}\text { Uniform } \\
\text { distribution }\end{array}$ & $\begin{array}{l}\text { Triangular } \\
\text { distribution }\end{array}$ & $\begin{array}{l}\text { Double } \\
\text { triangular }\end{array}$ & $\begin{array}{l}\text { Beta } \\
\text { distribution }\end{array}$ \\
\hline$i^{*}$ & 3 & 3 & 3 & 3 \\
$Q_{v}^{*}$ (units) & 21.90 & 21.88 & 21.85 & 24.53 \\
$p_{\text {on }}^{*}(\$)$ & 40.43 & 40.43 & 40.43 & 40.43 \\
$p_{\text {off }}^{*}(\$)$ & 58.39 & 58.39 & 58.39 & 58.39 \\
$P_{\xi}^{*}$ & 0.0000002 & 0.0000002 & 0.0000002 & 0.0000002 \\
$T P_{m}(\$)$ & 1254.57 & 1254.38 & 1254.12 & 1277.94 \\
\hline
\end{tabular}

TABLE 4. Optimum values for centralized case.

\begin{tabular}{lllll}
\hline \hline & $\begin{array}{l}\text { Uniform } \\
\text { distribution }\end{array}$ & $\begin{array}{l}\text { Triangular } \\
\text { distribution }\end{array}$ & $\begin{array}{l}\text { Double } \\
\text { triangular }\end{array}$ & $\begin{array}{l}\text { Beta } \\
\text { distribution }\end{array}$ \\
\hline$i^{*}$ & 3 & 3 & 3 & 3 \\
$Q_{v}^{*}($ units $)$ & 35.05 & 35.04 & 35.02 & 36.87 \\
$p_{\text {on }}^{*}(\$)$ & 47.67 & 47.69 & 47.71 & 45.94 \\
$p_{\text {off }}^{*}(\$)$ & 66.01 & 66.03 & 66.05 & 63.61 \\
$P_{\xi}^{*}$ & $1.379 \times 10^{-7}$ & $1.381 \times 10^{-7}$ & $1.382 \times 10^{-7}$ & $1.253 \times 10^{-7}$ \\
$L^{*}($ weeks $)$ & 3 & 3 & 3 & 3 \\
$R_{k}^{*}$ & 8.25 & 8.25 & 8.23 & 8.27 \\
$O_{c r}^{*}(\$)$ & 205.94 & 205.94 & 205.93 & 206.84 \\
$T P(\$)$ & 1904.08 & 1904.00 & 1904.17 & 1914.10 \\
\hline
\end{tabular}

\subsection{Result for centralize case}

Similarly for the centralize case, parametric values are obtained from Sarkar et al. [52] as follows: $A_{f}=3000$, $\rho=4000, \gamma=1.3, \delta=1.2, P_{u p}=\$ 0.2 /$ unit, $R_{h}=\$ 1.6 /$ unit, $\mu=2, O_{c_{0}}=\$ 250, g=90, \alpha_{1}=0.02, \alpha_{2}=0.003$, $A=\$ 30, T_{D}=\$ 2 /$ unit, $\psi=\$ 5 /$ unit, $S_{A}=\$ 3 /$ unit, $b=0.01, C_{w d}=\$ 0.5 /$ unit, $C_{p h}=\$ 2 /$ unit, $C_{v w}=$ $\$ 10 /$ unit, $F_{c c v}=\$ 3 /$ unit, $C_{l w}=\$ 2 /$ unit, $R_{P}=150$ unit, $P_{c v}=\$ 2.6 /$ unit, $M_{h}=\$ 4.6 /$ unit, $V_{c c v}=\$ 8 /$ unit, $T_{F}=\$ 2 /$ unit, $T_{V}=\$ 0.5 /$ unit, $P_{\theta_{0}}=0.0001$. The value related to Uniform distribution are $(0.03,0.07)$; related to Triangular distribution are $(0.04,0.07,0.05)$; and related to Beta distribution are $(0.03,0.07)$. The optimal result is provided in Table 4. From Table 4, it is clear that the profit is optimum when defective rate follows a Beta distribution and the profit is $\$ 1914.10$. From Table 4, one can find that the optimum selling price of the online channel is $45.94 \$ /$ unit and for offline channel is $63.61 \$ /$ unit, where as optimum ordered quantity is 36.87 units. Simultaneously, optimum ordering cost is $\$ 206.84$ and optimum safety stock is 8 unit. From the optimum Table 4 , the total system profit when defective rate follows an uniform distribution is $\$ 1904.08$, and the optimum selling price of the online channel is $47.67 \$ /$ unit and for offline channel is $66.01 \$ /$ unit, where as optimum ordered quantity is 35.05 units. Simultaneously, optimum ordering cost is $\$ 205.94$ and optimum safety stock is 8.25 unit. Similarly, the optimum system profit for triangular distribution is $\$ 1904.00$ and for double triangular distribution is $\$ 1904.17$. Figure 4 show the concavity graphically based on the decision variables. From the above examples it is clear centralize case is more beneficial due to the co ordination between the players. Also one can conclude that best result is obtain under the consideration of Bete distributed defective rate.

\subsection{Discussion and comparison}

In this section, some discussion regarding existing literature are presented as follows: 


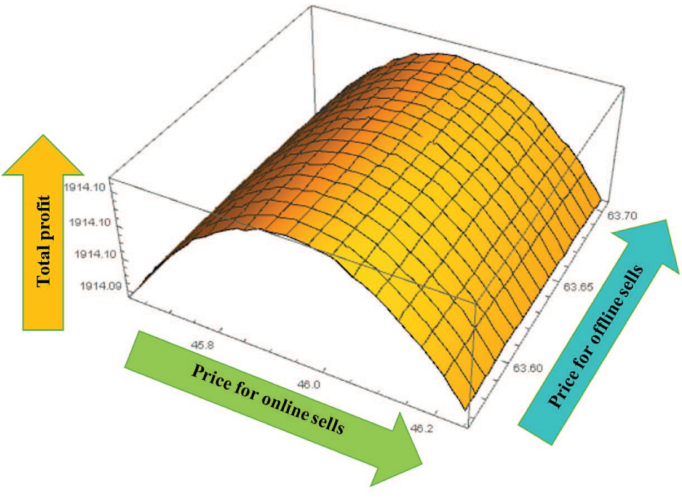

(a)

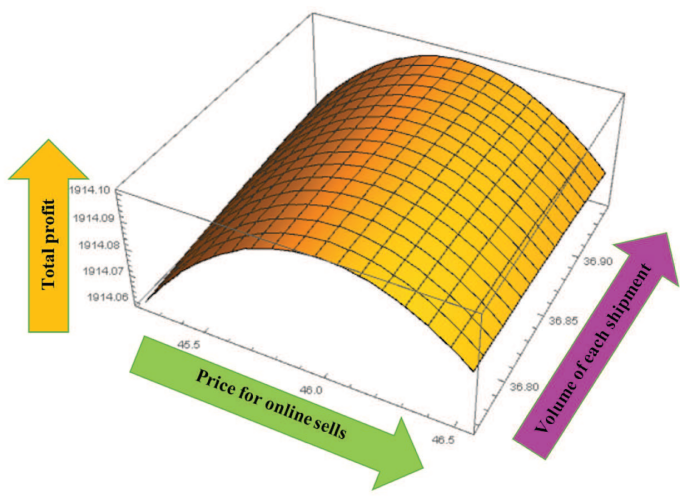

(b)

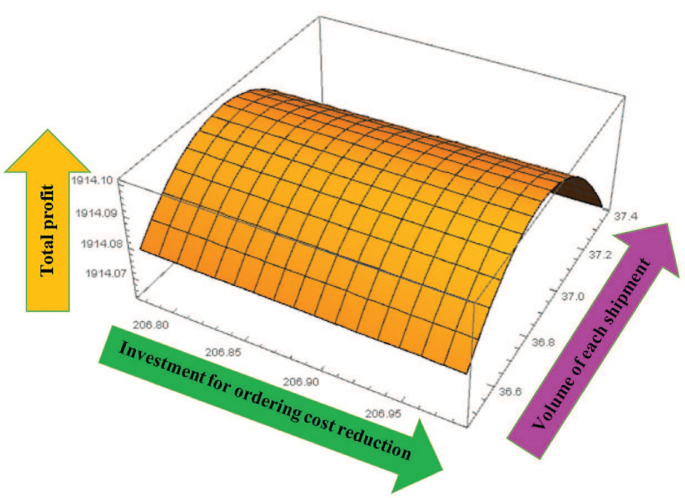

(c)

Figure 4. Concavity with respect to different decision variables when defective rate follows bete distribution. (a) Concavity with respect to two selling price. (b) Concavity with respect to online selling price and quantity. (c) Concavity with respect to investment for ordering cost reduction and quantity.

Sana and Chaudhuri [49] presented an EOQ model where demand varies with selling price, without O2O environment and lead time consideration. The profit for Sana and Chaudhuri [49] model was $\$ 91.54$, whereas price of the product was $\$ 49.57$ per unit. In the similar direct a SC model for green product and non-green product was developed by Sana [48] under the consideration of social responsibilities. However, he ignored O2O supply chain strategy and controllable lead time for his model. Moreover, he also examined his model for crisp data set value. The profit for non-green product in his model was $\$ 168.05$. The optimum selling price for non-green product was $\$ 63.71$ per unit. Compared to both the cases current study was more beneficial owing to the use of $\mathrm{O} 2 \mathrm{O}$ service strategy. By considering fuzzy uncertain environment and using triangular fuzzy number, Panda et al. [43] developed a supply chain model for single vendor and multiple retailer. They considered that demand was depend on selling price and on the stock. However, they ignored $\mathrm{O} 2 \mathrm{O}$ channeling strategy, controllable lead time, and backorder situation. The optimum system profit in Panda et al. [43] model was $\$ 67.51$. Thus, from the above illustration, it is clear that current study provides more better result in $\mathrm{O} 2 \mathrm{O}$ environment. The optimum profit of different existing study is provided in the following Table 5. The graphical representation in Figure 5 show the optimality of the current study compared to the existing literature. 
TABLE 5. Comparison table.

\begin{tabular}{llll}
\hline \hline$[49]$ & {$[43]$} & {$[48]$} & This study \\
\hline$\$ 91.54$ & $\$ 67.51$ & $\$ 168.05$ & $\$ 1914.10$ \\
\hline
\end{tabular}

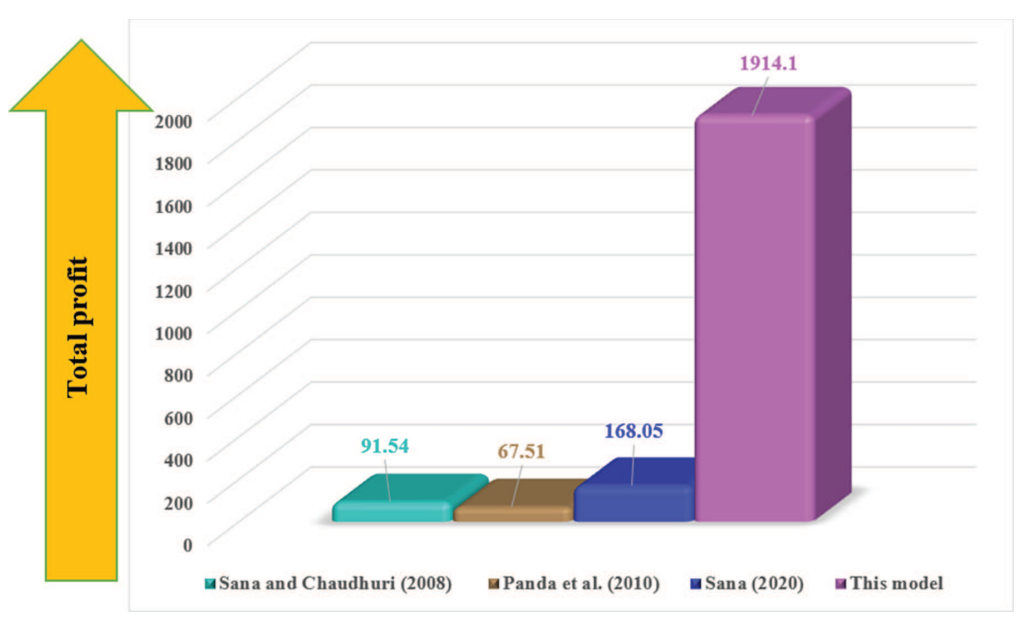

FiguRE 5. Graphical diagram of comparison.

\section{SEnsitivity ANALYSiS}

In this segment, sensitiveness of the critical parameters are illustrated. The rate of change in the value are considered as $(-50 \%,-25 \%,+25 \%,+50 \%)$. The change in total system profit for both crisp data set and fuzzy data set numerically provided in Table 6 . The effectiveness of the parameters in total system profit is graphically presented in Figure 6.

Now, from the Table 6, one can conclude as follows:

(i) The scaling parameter related to online demand is very much effective in determining the total system profit. About $\pm 85 \%$ change in total profit, if one change the scaling parameter related to online demand within the range $\pm 50 \%$. Thus, the parameter related to online demand is too much sensitive.

(ii) Similarly, the scaling parameter related to offline demand is also quite sensitive, change in the scaling parameter related to offline demand within the range $\pm 50 \%$, the total system profit will change about $\pm 55 \%$.

(iii) Unit holding cost for manufacturer is also quite sensitive. If one reduce the unit holding cost for manufacturer upto $50 \%$, then total system cost increases upto $34 \%$, whereas, increase in the unit holding cost for manufacturer upto $50 \%$, with increase total system profit decrease upto $34 \%$.

(iv) Similarly, the unit holding cost for retailer less sensitive compared to manufacturer's unit holding cost. It is quite natural that manufacturer needs huge space to keep the perfect as well as imperfect products. Moreover, due to SSMD policy manufacturer's unit holding cost is quite more sensitive compare to retailer's unit holding cost. Total system profit and unit holding cost for retailer inversely proportional. If one reduce the unit holding cost for retailer upto $50 \%$, then total system cost increases upto $11 \%$, whereas, increase in the unit holding cost for retailer upto $50 \%$, with increase total system profit decrease upto $11 \%$.

(v) From this model it is also clear that ordering cost plays a vital role in determining the system profit. Initial ordering cost is quite sensitive to archive the optimized profit. If one reduce the initial ordering 


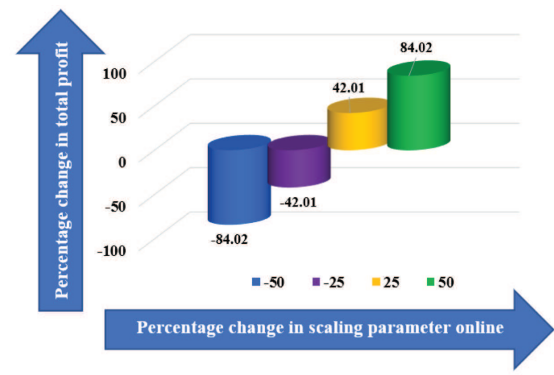

(a)

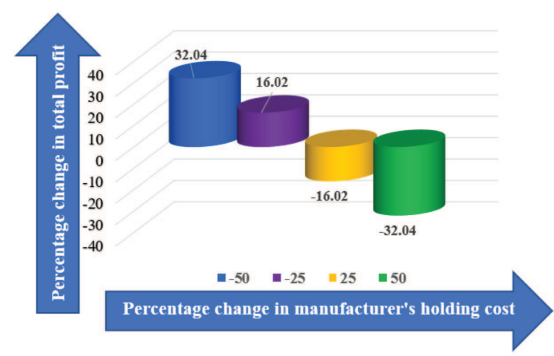

(c)

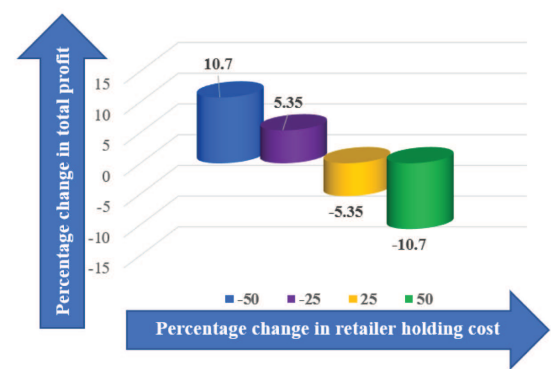

(e)

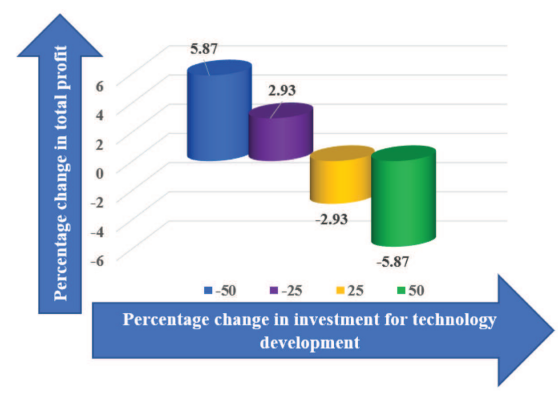

(g)

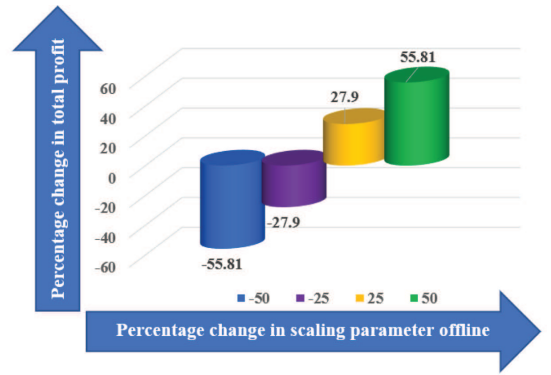

(b)

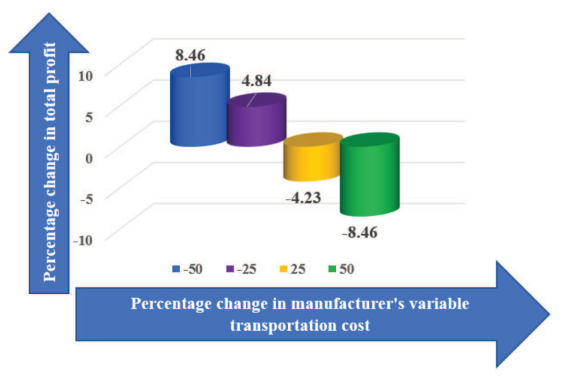

(d)

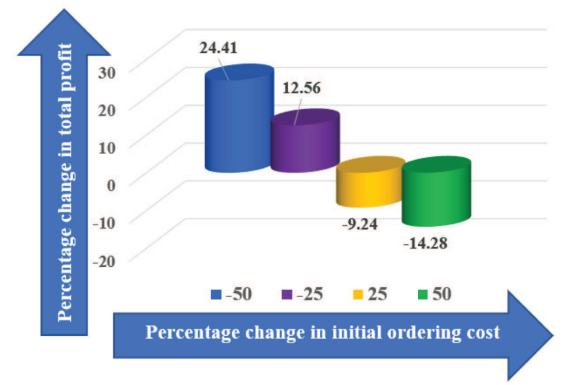

(f)

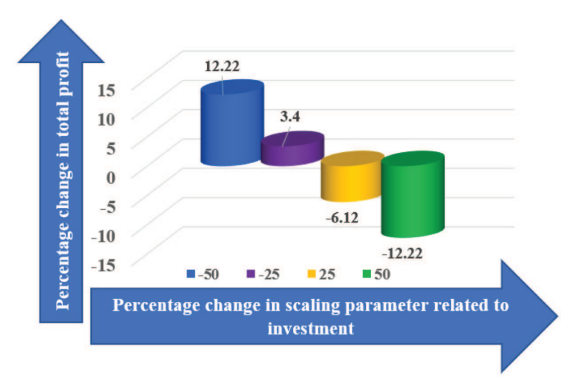

(h)

FIGURE 6. Effect of critical parameter on total system profit when defective rate follows Beta distribution. (a) Effect of scaling parameter related to online price. (b) Effect of scaling parameter related to offline price. (c) Effect of manufacturer's holding cost. (d) Effect of manufacturer's variable transportation cost. (e) Effect of retailer's holding cost. (f) Effect of initial ordering cost. (g) Effect of investment on technology development. (h) Effect of scaling parameter related to investment. 
TABLE 6. Sensitivity analysis table.

\begin{tabular}{|c|c|c|c|c|c|}
\hline Parameters & $\begin{array}{l}\text { Changes } \\
(\text { in } \%)\end{array}$ & $\begin{array}{l}\text { Uniform } \\
\text { distribution }\end{array}$ & $\begin{array}{l}\text { Triangular } \\
\text { distribution }\end{array}$ & $\begin{array}{l}\text { Double } \\
\text { triangular }\end{array}$ & $\begin{array}{l}\text { Beta } \\
\text { distribution }\end{array}$ \\
\hline \multirow[t]{4}{*}{$A_{f}$} & -50 & -82.50 & -82.79 & -82.42 & -84.02 \\
\hline & -25 & -41.25 & -41.40 & -41.21 & -42.01 \\
\hline & +25 & +41.25 & +41.40 & +41.21 & +42.01 \\
\hline & +50 & +82.50 & +82.79 & +82.42 & +84.02 \\
\hline \multirow[t]{4}{*}{$\rho$} & -50 & -55.00 & -55.15 & -54.96 & -55.81 \\
\hline & -25 & -27.50 & -27.58 & -27.48 & -27.90 \\
\hline & +25 & +27.51 & +27.58 & +27.48 & +27.90 \\
\hline & +50 & +55.00 & +55.15 & +54.96 & +55.81 \\
\hline \multirow[t]{4}{*}{$R_{h}$} & -50 & +10.63 & +10.63 & +10.62 & +10.70 \\
\hline & -25 & +5.31 & +5.32 & +5.31 & +5.35 \\
\hline & +25 & -5.31 & -5.32 & -5.31 & -5.35 \\
\hline & +50 & -10.63 & -10.63 & -10.62 & -10.70 \\
\hline \multirow[t]{4}{*}{$O_{c r_{0}}$} & -50 & +24.98 & +24.84 & +25.01 & +24.41 \\
\hline & -25 & +10.36 & +10.31 & +10.38 & +12.56 \\
\hline & +25 & -8.04 & -8.00 & -8.05 & -9.24 \\
\hline & +50 & -14.61 & -14.53 & -14.63 & -14.28 \\
\hline \multirow[t]{4}{*}{$A$} & -50 & +6.00 & +5.97 & +6.01 & +5.87 \\
\hline & -25 & +3.00 & +2.99 & +3.01 & +2.93 \\
\hline & +25 & -3.00 & -2.99 & -3.01 & -2.93 \\
\hline & +50 & -6.00 & -5.97 & -6.01 & -5.87 \\
\hline \multirow[t]{4}{*}{$M_{h}$} & -50 & +33.92 & +33.51 & +34.04 & +32.04 \\
\hline & -25 & +16.96 & +16.75 & +17.02 & +16.02 \\
\hline & +25 & -16.96 & -16.75 & -17.02 & -16.02 \\
\hline & +50 & -33.92 & -33.51 & -34.04 & -32.04 \\
\hline \multirow[t]{4}{*}{$g$} & -50 & +12.25 & +12.25 & +12.26 & +12.22 \\
\hline & -25 & +6.13 & +6.12 & +6.13 & +3.40 \\
\hline & +25 & -6.13 & -6.12 & -6.13 & -6.12 \\
\hline & +50 & -12.25 & -12.25 & -12.26 & -12.22 \\
\hline \multirow[t]{4}{*}{$T_{v}$} & -50 & +8.01 & +8.10 & +7.98 & +8.46 \\
\hline & -25 & +4.00 & +4.05 & +3.99 & +4.84 \\
\hline & +25 & -4.00 & -4.05 & -3.99 & -4.23 \\
\hline & +50 & -8.01 & -8.10 & -7.98 & -8.46 \\
\hline
\end{tabular}

cost upto $50 \%$, then total system cost increases upto $25 \%$, whereas, increase in the initial ordering cost upto $50 \%$, with increase total system profit decrease upto $15 \%$.

(vi) The scaling parameter related to investment function for ordering cost reduction is also quite sensitive. Since, ordering cost plays a vital role, thus scaling parameter related to investment function is also sensitive in optimizing the system profit. Decreasing in this scaling parameter increase total system profit. If one reduce the value of this scaling parameter upto $50 \%$, then total system cost increases upto $12 \%$, whereas, increase in the value of this scaling parameter upto $50 \%$, with increase total system profit decrease upto $12 \%$.

(vii) Under the SSMD transportation policy, the variable unit transportation cost for the manufacturer is little bit sensitive. Due to multiple delivery, the cost of transportation generally increase for the manufacturer. If one reduce the value of unit variable transportation cost upto $50 \%$, then total system cost increases upto $8 \%$, whereas, increase in the value of unit variable transportation cost upto $50 \%$, with increase total system profit decrease upto $8 \%$. 
(viii) Investment in the technology development is also quite sensitive. To provide better service to the customer, it is necessary to invest in the development in technology. If one reduce investment for technology development upto $50 \%$, then total system cost increases upto $6 \%$, whereas, increase in the investment for technology development upto $50 \%$, with increase total system profit decrease upto $6 \%$.

(ix) Rest parameters and unit costs are little bit sensitive in optimizing the total system profit.

\section{MANAGERIAL INSIGHTS}

For enhance the industry, the following the recommendations are taken:

(i) The defective O2O SCM is presented, where the total collaborative profit is minimized based on ideal value of decision variables. To minimize defective generation, the manager may sustain the investment quality enhancement by this research. It is basic for dependable SCM frameworks. The manager may minimize the total cost with the help of utilizing the numerical result as well as provide best service to their purchaser.

(ii) To make high popularity of the products and control the market selling price, depended online and offline demand are considered. Both the demand services for both the channel are improved, paralley, investment for improving the technology directly help to supply superior benefit to the customers.

(iii) A uninterrupted investment was implied in reducing the ordering cost of the retailer, which straight away enhance the process total profit, as well as better service, can be provided to the supply chain player.

(iv) Another important finding is the variable safety factor. The holding cost is controlled by variable safety factor depending on fidgeting demand of market. The holding cost is minimized by restricted stock, when demand is low, on the other hand, the holding cost is raised in stocking the products, when the demand is enormous. The managers may attention on it of the production system. Moreover, by controlling the stock out the situation, managers can provide better service to their customers.

(v) The industry manager can supply superior benefit to their customers through "variable lead time". Because reduced lead time controls customer satisfaction as well as fulfills the market demand, which directly enhances the service label of the supply chain.

(vi) Imperfect materials increase the whole cost and minimize the whole profit. To minimize such a loss, the managers of the production system may be concerned to provide the best service to their customers.

\section{Conclusions}

An intelligent dual channel SCM with reduced lead time and upgraded service in an uncertain environment is developed in this study. To provide outstanding service and enhance SCM profit, it is required to determine the exact demand for a particular product. For more applicability of this study in real world, demand for the product is vary with selling price. Owing to the tendency of e-marketing, this study contemplates different selling prices for a single product for various channels. This study contemplates two separate channels, namely online and offline, where the retailer uses only one channel to parches the product from the manufacturer, but sell in both the channel with two different selling prices.

Typically, the offline channel's selling price is quite expensive compared to online channels due to different issues like labor, energy, and many more. However, some customers still believe in visiting the retail shop and prefer to choose the product offline. Trade-off between the players of the supply chain always beneficial, which is proved by centralize and decentralize cases. In any $\mathrm{O} 2 \mathrm{O}$ supply chain, placing an order plays a vital role. When to place an order and how much to order are also very important for any supply chain. Backorder and safety stock also play a critical role in deciding when to order and how much to order? Thus, in this study, exact backorder is determine with the help of marginal value and variable safety factors. The use of SSMD transportation policy is one of "the limitations of this model", and one can use single-setup-multi-unequal-delivery (SSMUD) transportation, which is more beneficial. Instated of continuous investment, one can use the discrete investment for ordering cost reduction [50]. Some intelligent techniques such as autonomation can identify defective products [13]. To provide optimal service manufacturer can use a third party logistic (3PL) system [11]. Moreover, one 
can expand this study by introducing the production rate dependent defective rate. By considering credit-period [59] one can extend the current study in a new direction. Fast Evolutionary Algorithm [31] can be use in future to obtain the optimal result of the supply chain. Innovative drone technology can be used for the delivery of the product online. Also, by providing different services (unpaid, partially paid, full paid) company can enhance its profit, which will be exciting research in the feature. Instated of a single product, one can extend this study for multiple assembled green products.

\section{Appendix A.}

Total profit $\left(Q_{v}, i, L, p_{\text {on }}, p_{\text {off }}, P_{\xi}, O_{c r}, R_{k}\right)$ is combined $\Theta$

$$
\begin{aligned}
& \frac{\partial \Theta(\cdot)}{\partial Q}=\frac{\left(A_{f} p_{\mathrm{on}}^{-\gamma}+\rho p_{\mathrm{off}}^{-\delta}\right)}{Q_{v}^{2}}\left[O_{c r}+T_{D}+T_{F}+F_{c c v}+\frac{C_{w d}+C_{p h}+C_{v w}+C_{l w}+S_{A}}{i}\right. \\
& \left.+\frac{2 \psi \sigma_{x}^{2}}{3 Q_{v}}\left(\Phi^{2}\left(R_{k}\right)-\Phi^{2}\left(R_{k}+\frac{Q_{v}}{\sigma_{x}}\right)\right)\right]+\frac{\sigma_{x}^{2} R_{h}}{Q_{v}^{2}}\left(\Phi^{2}\left(R_{k}\right)-\Phi^{2}\left(R_{k}+\frac{Q_{v}}{\sigma_{x}}\right)\right) \\
& +\frac{\sigma_{x}}{Q_{v}}\left[\left(A_{f} p_{\text {on }}^{-\gamma}+\rho p_{\text {off }}^{-\delta}\right) \psi+1\right] \Phi^{1}\left(R_{k}+\frac{Q_{v}}{\sigma_{x}}\right)-\frac{1}{2}\left[i S_{c}\left(A_{f} p_{\text {on }}^{-\gamma}+\rho p_{\text {off }}^{-\delta}\right) P_{\xi}\right. \\
& \left.+M_{h}\left[1+(i-2)\left(1-\frac{\left(A_{f} p_{\text {on }}^{-\gamma}+\rho p_{\text {off }}^{-\delta}\right)}{(1-\omega) R_{P}}\right)\right]\right] \\
& \frac{\partial \Theta(\cdot)}{\partial L}=-\left[R_{h}\left(\mu+R_{k} \frac{\partial \sigma_{x}}{\partial L}\right)+\left(R_{h}+\frac{\left(A_{f} p_{\mathrm{on}}^{-\gamma}+\rho p_{\mathrm{off}}^{-\delta}\right)}{Q_{v}}\right)\left[\left(\Phi^{2}\left(R_{k}\right)-\Phi^{2}\left(R_{k}+\frac{Q_{v}}{\sigma_{x}}\right)\right) \frac{2 \sigma_{x}}{Q_{v}} \frac{\partial \sigma_{x}}{\partial L}\right.\right. \\
& \left.\left.+\Phi^{1}\left(R_{k}+\frac{Q_{v}}{\sigma_{x}}\right) \frac{\partial \sigma_{x}}{\partial L}\right]\right] \\
& \frac{\partial \Theta(\cdot)}{\partial R_{k}}=-\left[R_{h} \sigma_{x}+\left(\Phi^{1}\left(R_{k}\right)-\Phi^{1}\left(R_{k}+\frac{Q_{v}}{\sigma_{x}}\right)\right) \frac{\sigma_{x}}{Q_{v}}\left[R_{h} \sigma_{x}+\frac{\left(A_{f} p_{\text {on }^{-\gamma}}+\rho p_{\text {off }}{ }^{-\delta}\right) \psi \sigma_{x}}{Q_{v}}\right]\right] \\
& \frac{\partial \Theta(\cdot)}{\partial p_{\text {on }}}=A_{f} p_{\text {on }}^{-\gamma}+A_{f} \gamma p_{\text {on }}^{-1-\gamma}\left[T_{V}+V_{c c v}-\left(p_{\text {on }}-C_{u p}\right)+\frac{T_{F}+F_{c c v}+T_{D}+O_{c r}}{Q_{v}}\right. \\
& \left.+\frac{C_{w d}+C_{p h}+C_{v w}+C_{l w}+S_{A}}{i Q_{v}}-\frac{(i-2) M_{h}}{2(1-\omega) R_{p}}+\frac{\sigma_{x}^{2} \psi}{Q_{v}^{2}}\left(\Phi^{2}(K)-\Phi^{2}\left(R_{k}+\frac{Q_{v}}{\sigma_{x}}\right)\right)\right] \\
& \frac{\partial \Theta(\cdot)}{\partial p_{\text {off }}}=\rho p_{\text {off }}^{-\delta}+\rho \delta p_{\text {off }}^{-1-\delta}\left[T_{V}+V_{c c v}-\left(p_{\text {off }}-C_{u p}\right)+\frac{T_{F}+F_{c c v}+T_{D}+O_{c r}}{Q_{v}}\right. \\
& \left.+\frac{C_{w d}+C_{p h}+C_{v w}+C_{l w}+S_{A}}{i Q_{v}}-\frac{(i-2) M_{h}}{2(1-\omega) R_{p}}+\frac{\sigma_{x}^{2} \psi}{Q_{v}^{2}}\left(\Phi^{2}(K)-\Phi^{2}\left(R_{k}+\frac{Q_{v}}{\sigma_{x}}\right)\right)\right] \\
& \frac{\partial \Theta(\cdot)}{\partial P_{\xi}}=\frac{b}{P_{\xi}}-\frac{i S_{c} Q_{v}\left(A_{f} p_{\text {on }}^{-\gamma}+\rho p_{\text {off }}^{-\delta}\right)}{2} ; \quad \frac{\partial \Theta(\cdot)}{\partial O_{c r}}=-\frac{\left(A_{f} p_{\text {on }}^{-\gamma}+\rho p_{\text {off }}^{-\delta}\right)}{Q_{v}}+\frac{g}{O_{c r}} .
\end{aligned}
$$




\section{Appendix B.}

Here

$$
\begin{aligned}
& \sigma_{x}^{2}=L \sigma^{2}+\mu^{2} \sigma_{L}^{2} ; \quad \frac{\partial \sigma_{x}}{\partial L}=\frac{\sigma^{2}}{2 \sqrt{L \sigma^{2}+\mu^{2} \sigma_{L}^{2}}} ; \quad \frac{\partial^{2} \sigma_{x}}{\partial L^{2}}=-\frac{\sigma^{2}\left(\sigma^{2}+2 \mu^{2} \sigma_{L} \frac{\partial \sigma_{L}}{\partial L}\right)}{4\left(L \sigma^{2}+\mu^{2} \sigma_{L}^{2}\right)^{\frac{3}{2}}} \\
& \Pi_{1}=\left[T_{V}+V_{c c v}+C_{u p}+\frac{T_{F}+F_{c c v}+T_{D}+O_{c r}}{Q_{v}}+\frac{C_{w d}+C_{p h}+C_{v w}+C_{l w}+S_{A}}{i Q_{v}}\right. \\
& \left.-\frac{(i-2) M_{h}}{2(1-\omega) R_{p}}+\frac{\sigma_{x}^{2} \psi}{Q_{v}^{2}}\left(\Phi^{2}(K)-\Phi^{2}\left(R_{k}+\frac{Q_{v}}{\sigma_{x}}\right)\right)\right] \\
& \Pi_{2}=\frac{\left(A_{f} p_{\mathrm{on}}^{-\gamma}+\rho p_{\mathrm{off}}^{-\delta}\right)}{Q_{v}^{2}}\left[O_{c r}+T_{D}+T_{F}+F_{c c v}+\frac{C_{w d}+C_{p h}+C_{v w}+C_{l w}+S_{A}}{i}\right] \\
& \Pi_{3}=\frac{\left(\Phi^{2}\left(R_{k}\right)-\Phi^{2}\left(R_{k}+\frac{Q_{v}}{\sigma_{x}}\right)\right)}{Q_{v}^{2}}\left[\sigma_{x} R_{h}+\frac{2 \sigma_{x}^{2} \psi}{3 Q_{v}}\right] \\
& \Pi_{4}=\frac{\sigma_{x}}{Q_{v}}\left[\left(A_{f} p_{\text {on }}^{-\gamma}+\rho p_{\text {off }}^{-\delta}\right) \psi+1\right] \Phi^{1}\left(R_{k}+\frac{Q_{v}}{\sigma_{x}}\right) \\
& \Pi_{5}=\frac{1}{2}\left[i S_{c}\left(A_{f} p_{\mathrm{on}}^{-\gamma}+\rho p_{\mathrm{off}}^{-\delta}\right) P_{\xi}+M_{h}\left[1+(i-2)\left(1-\frac{\left(A_{f} p_{\mathrm{on}}^{-\gamma}+\rho p_{\text {off }}^{-\delta}\right)}{(1-\omega) R_{P}}\right)\right]\right] .
\end{aligned}
$$

\section{Appendix C.}

$$
\begin{aligned}
\frac{\partial^{2} \Theta(\cdot)}{\partial Q_{v}^{2}}= & -\left[\frac { 2 ( A _ { f } p _ { \text { on } } ^ { - \gamma } + \rho p _ { \text { off } } ^ { - \delta } ) } { Q _ { v } ^ { 3 } } \left[O_{c r}+T_{D}+T_{F}+F_{c c v}+\frac{C_{w d}+C_{p h}+C_{v w}+C_{l w}+S_{A}}{i}\right.\right. \\
& \left.+\frac{2 \psi \sigma_{x}^{2}}{3 Q_{v}^{2}}\left(\Phi^{2}\left(R_{k}\right)-\Phi^{2}\left(R_{k}+\frac{Q_{v}}{\sigma_{x}}\right)\right)\right]+\frac{2 \sigma_{x}^{2} R_{h}}{Q_{v}^{3}}\left(\Phi^{2}\left(R_{k}\right)-\Phi^{2}\left(R_{k}+\frac{Q_{v}}{\sigma_{x}}\right)\right) \\
& \left.+\frac{\sigma_{x}}{Q_{v}^{2}}\left[\left(A_{f} p_{\text {on }}^{-\gamma}+\rho p_{\text {off }}^{-\delta}\right) \psi+1\right] \Phi^{1}\left(R_{k}+\frac{Q_{v}}{\sigma_{x}}\right)\right]=-\Gamma_{1}(\text { say })<0 \\
\frac{\partial^{2} \Theta(\cdot)}{\partial R_{k}^{2}}= & -\left[\frac{\sigma_{x}}{Q_{v}}\left[\sigma_{x} R_{h}+\frac{\sigma_{x} \psi\left(A_{f} p_{\text {on }}^{-\gamma}+\rho p_{\text {off }}^{-\delta}\right.}{Q_{v}^{2}}\right]\left(\Phi\left(R_{k}\right)-\Phi\left(R_{k}+\frac{Q_{v}}{\sigma_{x}}\right)\right)\right]=-\Gamma_{2}(\text { say })<0 \\
\frac{\partial^{2} \Theta(\cdot)}{\partial L^{2}}= & -\left[R_{h} R_{k} \frac{\partial^{2} \sigma_{x}}{\partial L^{2}}+\left(R_{h}+\frac{\left(A_{f} p_{\text {on }}^{-\gamma}+\rho p_{\text {off }}^{-\delta}\right)}{Q_{v}}\right)\left[\left(\frac{2}{\sigma_{x}}\left(\frac{\partial \sigma_{x}}{\partial L}\right)^{2}+\frac{\partial^{2} \sigma_{x}}{\partial L^{2}}\right) \Phi^{1}\left(R_{k}+\frac{Q_{v}}{\sigma_{X}}\right)\right.\right. \\
& +\left(\Phi^{2}\left(R_{k}\right)-\Phi^{2}\left(R_{k}+\frac{Q_{v}}{\sigma_{x}}\right)\right)\left(\frac{2}{Q_{v}}\left(\frac{\partial \sigma_{x}}{\partial L}\right)^{2}+\frac{2 \sigma_{x}}{Q_{v}} \frac{\partial^{2} \sigma_{x}}{\partial L^{2}}\right. \\
& \left.\left.\left.-\Phi\left(R_{k}+\frac{Q_{v}}{\sigma_{x}}\right) \frac{Q_{v}}{\sigma_{x}^{2}}\left(\frac{\partial \sigma_{x}}{\partial L}\right)^{2}\right)\right]\right]=-\Gamma_{3}(\text { say })<0
\end{aligned}
$$


INTELLIGENT SERVICING STRATEGY FOR AN ONLINE-TO-OFFLINE (O2O) SUPPLY CHAIN

$$
\begin{aligned}
& \frac{\partial^{2} \Theta(\cdot)}{\partial p_{\mathrm{on}}^{2}}=-\left[2 A_{f} \gamma p_{\mathrm{on}}^{-\gamma-1}+A_{f} \gamma(\gamma+1) p_{\mathrm{on}}^{-2-\gamma}\left[T_{V}+V_{c c v}-\left(p_{\mathrm{on}}-C_{u p}\right)+\frac{T_{F}+F_{c c v}+T_{D}+O_{c r}}{Q_{v}}\right.\right. \\
& +\frac{C_{w d}+C_{p h}+C_{v w}+C_{l w}+S_{A}}{i Q_{v}}-\frac{(i-2) M_{h}}{2(1-\omega) R_{p}} \\
& \left.\left.+\frac{\sigma_{x}^{2} \psi}{Q_{v}^{2}}\left(\Phi^{2}(K)-\Phi^{2}\left(R_{k}+\frac{Q_{v}}{\sigma_{x}}\right)\right)\right]\right]=-\Gamma_{4}(\text { say })<0 \\
& \frac{\partial^{2} \Theta(\cdot)}{\partial p_{\mathrm{off}}^{2}}=-\left[2 \rho \delta p_{\mathrm{off}}^{-\delta-1}+\rho \delta(\delta+1) p_{\mathrm{off}}^{-2-\delta}\left[T_{V}+V_{c c v}-\left(p_{\mathrm{off}}-C_{u p}\right)+\frac{T_{F}+F_{c c v}+T_{D}+O_{c r}}{Q_{v}}\right.\right. \\
& +\frac{C_{w d}+C_{p h}+C_{v w}+C_{l w}+S_{A}}{i Q_{v}}-\frac{(i-2) M_{h}}{2(1-\omega) R_{p}} \\
& \left.\left.+\frac{\sigma_{x}^{2} \psi}{Q_{v}^{2}}\left(\Phi^{2}(K)-\Phi^{2}\left(R_{k}+\frac{Q_{v}}{\sigma_{x}}\right)\right)\right]\right]-\Gamma_{5} \text { (say) }<0 \\
& \frac{\partial^{2} \Theta(\cdot)}{\partial P_{\xi}^{2}}=-\frac{b}{P_{\xi}^{2}}<0 ; \quad \frac{\partial^{2} T P(\cdot)}{\partial O_{c r}^{2}}=-\frac{g}{O_{c r}^{2}}<0 \\
& \frac{\partial^{2} \Theta(\cdot)}{\partial Q_{v} \partial p_{\text {on }}}=\frac{\partial^{2} \Theta(\cdot)}{\partial p_{\text {on }} \partial Q_{v}}=-\left[\frac { ( A _ { f } \gamma p _ { \text { on } } ^ { - 1 - \gamma } ) } { Q _ { v } ^ { 2 } } \left[O_{c r}+T_{D}+T_{F}+F_{c c v}+\frac{C_{w d}+C_{p h}+C_{v w}+C_{l w}+S_{A}}{i}\right.\right. \\
& \left.+\frac{2 \psi \sigma_{x}^{2}}{3 Q_{v}}\left(\Phi^{2}\left(R_{k}\right)-\Phi^{2}\left(R_{k}+\frac{Q_{v}}{\sigma_{x}}\right)\right)\right]+\frac{\sigma_{x}^{2} R_{h}}{Q_{v}^{2}}\left(\Phi^{2}\left(R_{k}\right)-\Phi^{2}\left(R_{k}+\frac{Q_{v}}{\sigma_{x}}\right)\right) \\
& +\frac{\sigma_{x}}{Q_{v}}\left[A_{f} \gamma p_{\mathrm{on}}^{-1-\gamma} \psi+1\right] \Phi^{1}\left(R_{k}+\frac{Q_{v}}{\sigma_{x}}\right) \\
& \left.-\frac{1}{2}\left[i S_{c} A_{f} \gamma p_{\mathrm{on}}^{-1-\gamma} P_{\xi}+M_{h}\left[(i-2)\left(1-\frac{A_{f} \gamma p_{\mathrm{on}}^{-1-\gamma}}{(1-\omega) R_{P}}\right)\right]\right]\right]=\Theta_{1} \text { (say) } \\
& \frac{\partial^{2} \Theta(\cdot)}{\partial Q_{v} \partial p_{\text {off }}}=\frac{\partial^{2} \Theta(\cdot)}{\partial p_{\text {off }} \partial Q_{v}}=-\left[\frac { ( \rho \delta p _ { \text { off } } ^ { - 1 - \delta } ) } { Q _ { v } ^ { 2 } } \left[O_{c r}+T_{D}+T_{F}+F_{c c v}+\frac{C_{w d}+C_{p h}+C_{v w}+C_{l w}+S_{A}}{i}\right.\right. \\
& \left.+\frac{2 \psi \sigma_{x}^{2}}{3 Q_{v}}\left(\Phi^{2}\left(R_{k}\right)-\Phi^{2}\left(R_{k}+\frac{Q_{v}}{\sigma_{x}}\right)\right)\right]+\frac{\sigma_{x}^{2} R_{h}}{Q_{v}^{2}}\left(\Phi^{2}\left(R_{k}\right)-\Phi^{2}\left(R_{k}+\frac{Q_{v}}{\sigma_{x}}\right)\right) \\
& +\frac{\sigma_{x}}{Q_{v}}\left[\rho \delta p_{\mathrm{off}}^{-1-\delta} \psi+1\right] \Phi^{1}\left(R_{k}+\frac{Q_{v}}{\sigma_{x}}\right)-\frac{1}{2}\left[i S_{c} \rho \delta p_{\mathrm{off}}^{-1-\delta} P_{\xi}\right. \\
& \left.\left.+M_{h}\left[(i-2)\left(\frac{\rho \delta p_{\text {off }}^{-1-\delta}}{(1-\omega) R_{P}}\right)\right]\right]\right]=\Theta_{2} \text { (say) } \\
& \frac{\partial^{2} \Theta(\cdot)}{\partial Q_{v} \partial R_{k}}=\frac{\partial^{2} T P(\cdot)}{\partial R_{k} \partial Q_{v}}=\left(\Phi^{1}\left(R_{k}\right)-\Phi^{1}\left(R_{k}+\frac{Q_{v}}{\sigma_{x}}\right)\right) \frac{\sigma_{x}^{2}}{Q_{v}^{2}}\left(R_{h}+\frac{2\left(A_{f} p_{\text {on }}^{-\gamma}+\rho p_{\text {off }}^{-\delta}\right) \psi}{Q_{v}}\right) \\
& -\Phi\left(R_{k}+\frac{Q_{v}}{\sigma_{x}}\right) \frac{\sigma_{x}}{Q_{v}}\left(R_{h}+\frac{\left(A_{f} p_{\text {on }}^{-\gamma}+\rho p_{\text {off }}^{-\delta}\right) \psi}{Q_{v}}\right)=\Theta_{3} \text { (say) } \\
& \frac{\partial^{2} \Theta(\cdot)}{\partial Q_{v} \partial L}=\frac{\partial^{2} \Theta(\cdot)}{\partial L \partial Q_{v}}=\left(\Phi^{2}\left(R_{k}\right)-\Phi^{2}\left(R_{k}+\frac{Q_{v}}{\sigma_{x}}\right)\right) \frac{2 \sigma_{x}}{Q^{2}} \frac{\partial \sigma_{x}}{\partial L}\left[\frac{2\left(A_{f} p_{\text {on }}^{-\gamma}+\rho p_{\text {off }}^{-\delta}\right)}{i Q}+R_{h}\right]
\end{aligned}
$$




$$
\begin{aligned}
& -\Phi^{1}\left(R_{k}+\frac{Q_{v}}{\sigma_{x}}\right) \frac{\partial \sigma_{x}}{\partial L}\left[\frac{\left(a+\alpha \beta^{\xi}\right)}{i Q_{v}^{2}}+\frac{2}{Q_{v}}\left(R_{h}+\frac{\left(A_{f} p_{\text {on }}^{-\gamma}+\rho p_{\text {off }}^{-\delta}\right)}{i Q}\right)-\frac{1}{\sigma_{x}}\right]=\Theta_{4} \text { (say) } \\
& \frac{\partial^{2} \Theta(\cdot)}{\partial Q_{v} \partial O_{c r}}=\frac{\partial^{2} \Theta(\cdot)}{\partial O_{c r} \partial Q_{v}}=\frac{\left(A_{f} p_{\text {on }}^{-\gamma}+\rho p_{\text {off }}^{-\delta}\right)}{Q_{v}} ; \quad \frac{\partial^{2} \Theta(\cdot)}{\partial Q_{v} \partial P_{\xi}}=\frac{\partial^{2} \Theta(\cdot)}{\partial P_{\xi} \partial Q_{v}}=-\frac{1}{2} i S_{c}\left(A_{f} p_{\text {on }}^{-\gamma}+\rho p_{\text {off }}^{-\delta}\right) \\
& \frac{\partial^{2} \Theta(\cdot)}{\partial p_{\text {on }} \partial R_{k}}=\frac{\partial^{2} \Theta(\cdot)}{\partial R_{k} \partial p_{\text {on }}}=\left(\Phi^{1}\left(R_{k}\right)-\Phi^{1}\left(R_{k}+\frac{Q_{v}}{\sigma_{x}}\right)\right) \frac{A_{f} \gamma p_{\text {on }}{ }^{-1-\gamma} \psi \sigma_{x}^{2}}{Q_{v}^{2}}=\Theta_{5} \text { (say) } \\
& \frac{\partial^{2} \Theta(\cdot)}{\partial p_{\text {on }} \partial L}=\frac{\partial^{2} \Theta(\cdot)}{\partial L \partial p_{\text {on }}}=\frac{\left(A_{f} \gamma p_{\text {on }}{ }^{-1-\gamma}\right)}{Q_{v}}\left[\left(\Phi^{2}\left(R_{k}\right)-\Phi^{2}\left(R_{k}+\frac{Q_{v}}{\sigma_{x}}\right)\right) \frac{2 \sigma_{x}}{Q_{v}} \frac{\partial \sigma_{x}}{\partial L}\right. \\
& \left.+\Phi^{1}\left(R_{k}+\frac{Q_{v}}{\sigma_{x}}\right) \frac{\partial \sigma_{x}}{\partial L}\right]=\Theta_{6} \text { (say) } \\
& \frac{\partial^{2} \Theta(\cdot)}{\partial p_{\text {on }} \partial O_{c r}}=\frac{\partial^{2} \Theta(\cdot)}{\partial O_{c r} \partial p_{\text {on }}}=\frac{A_{f} p_{\text {on }}^{-1-\gamma}}{Q_{v}} ; \frac{\partial^{2} \Theta(\cdot)}{\partial p_{\text {on }} \partial P_{\xi}}=\frac{\partial^{2} \Theta(\cdot)}{\partial P_{\xi} \partial p_{\text {on }}}=\frac{A_{f} i Q_{v} S_{c} \gamma p_{\text {on }}^{-1-\gamma}}{2} ; \\
& \frac{\partial^{2} \Theta(\cdot)}{\partial p_{\text {on }} \partial p_{\text {off }}}=\frac{\partial^{2} \Theta(\cdot)}{\partial p_{\text {off }} \partial p_{\text {on }}}=0 \\
& \frac{\partial^{2} \Theta(\cdot)}{\partial p_{\text {off }} \partial R_{k}}=\frac{\partial^{2} \Theta(\cdot)}{\partial R_{k} \partial p_{\text {off }}}=\left(\Phi^{1}\left(R_{k}\right)-\Phi^{1}\left(R_{k}+\frac{Q_{v}}{\sigma_{x}}\right)\right) \frac{\rho \delta p_{\text {off }^{-1-\delta} \psi \sigma_{x}^{2}}}{Q_{v}^{2}}=\Theta_{7} \text { (say) } \\
& \frac{\partial^{2} \Theta(\cdot)}{\partial p_{\text {off }} \partial L}=\frac{\partial^{2} \Theta(\cdot)}{\partial L \partial p_{\text {off }}}=\frac{\left(\rho \delta p_{\text {off }^{-1-\delta}}\right)}{Q_{v}}\left[\left(\Phi^{2}\left(R_{k}\right)-\Phi^{2}\left(R_{k}+\frac{Q_{v}}{\sigma_{x}}\right)\right) \frac{2 \sigma_{x}}{Q_{v}} \frac{\partial \sigma_{x}}{\partial L}\right. \\
& \left.+\Phi^{1}\left(R_{k}+\frac{Q_{v}}{\sigma_{x}}\right) \frac{\partial \sigma_{x}}{\partial L}\right]=\Theta_{8} \text { (say) } \\
& \frac{\partial^{2} \Theta(\cdot)}{\partial p_{\text {off }} \partial O_{c r}}=\frac{\partial^{2} \Theta(\cdot)}{\partial O_{c r} \partial p_{\text {off }}}=\frac{\rho p_{\text {off }}^{-1-\delta}}{Q_{v}} ; \quad \frac{\partial^{2} \Theta(\cdot)}{\partial p_{\text {off }} \partial P_{\xi}}=\frac{\partial^{2} \Theta(\cdot)}{\partial P_{\xi} \partial p_{\text {off }}}=\frac{1}{2} \rho i Q_{v} S_{c} \delta p_{\text {off }}^{-1-\delta} \\
& \frac{\partial^{2} \Theta(\cdot)}{\partial L \partial R_{k}}=\frac{\partial^{2} \Theta(\cdot)}{\partial R_{k} \partial L}=-\left[R_{h} \frac{\partial \sigma_{x}}{\partial L}+\left(R_{h}+\frac{\left(A_{f} p_{\text {on }}^{-\gamma}+\rho p_{\text {off }}^{-\delta}\right)}{Q_{v}}\right) \frac{\partial \sigma_{x}}{\partial L}\left[\left(\Phi^{1}\left(R_{k}\right)-\Phi^{1}\left(R_{k}+\frac{Q_{v}}{\sigma_{x}}\right)\right) \frac{2 \sigma_{x}}{Q_{v}}\right.\right. \\
& \left.\left.+\Phi\left(R_{k}+\frac{Q_{v}}{\sigma_{x}}\right)\right]\right]=\Theta_{9} \text { (say) } \\
& \frac{\partial^{2} \Theta(\cdot)}{\partial L \partial O_{c r}}=\frac{\partial^{2} \Theta(\cdot)}{\partial O_{c r} \partial L}=0 ; \quad \frac{\partial^{2} \Theta(\cdot)}{\partial L \partial P_{\xi}}=\frac{\partial^{2} \Theta(\cdot)}{\partial P_{\xi} \partial L}=0 ; \quad \frac{\partial^{2} \Theta(\cdot)}{\partial R_{k} \partial O_{c r}}=\frac{\partial^{2} \Theta(\cdot)}{\partial O_{c r} \partial R_{k}}=0 \\
& \frac{\partial^{2} \Theta(\cdot)}{\partial R_{k} \partial P_{\xi}}=\frac{\partial^{2} \Theta(\cdot)}{\partial P_{\xi} \partial R_{k}}=0 ; \quad \frac{\partial^{2} \Theta(\cdot)}{\partial O_{c r} \partial P_{\xi}}=\frac{\partial^{2} \Theta(\cdot)}{\partial P_{\xi} \partial O_{c r}}=0 .
\end{aligned}
$$

\section{Appendix D.}

$$
\begin{aligned}
\left|H_{11}\right| & =\left|\frac{\partial^{2} \Theta(\cdot)}{\partial P_{\xi}^{2}}\right|=-\frac{b}{P_{\xi}^{2}}<0 ; \quad\left|H_{22}\right|=\left|\begin{array}{cc}
\frac{\partial^{2} \Theta(\cdot)}{\partial P_{\xi}^{2}} & \frac{\partial^{2} \Theta(\cdot)}{\partial O_{c r} \partial P_{\xi}} \\
\frac{\partial^{2} \Theta(\cdot)}{\partial O_{c r} \partial P_{\xi}} & \frac{\partial^{2} \Theta(\cdot)}{\partial O_{c r}^{2}}
\end{array}\right|=\frac{\partial^{2} \Theta(\cdot)}{\partial P_{\xi}^{2}} \frac{\partial^{2} \Theta(\cdot)}{\partial O_{c r}^{2}}=\frac{g b}{P_{\xi}^{2} O_{c r}^{2}}>0 \\
\left|H_{33}\right| & =\left|\begin{array}{ccc}
\frac{\partial^{2} \Theta(\cdot)}{\partial P_{\xi}^{2}} & \frac{\partial^{2} \Theta(\cdot)}{\partial P_{\xi} \partial O_{c r}} & \frac{\partial^{2} \Theta(\cdot)}{\partial P_{\xi} \partial R_{k}} \\
\frac{\partial^{2} \Theta(\cdot)}{\partial O_{c r} \partial P_{\xi}} & \frac{\partial^{2} \Theta(\cdot)}{\partial O_{c r}^{2}} & \frac{\partial^{2} \Theta(\cdot)}{\partial O_{c r} \partial R_{k}} \\
\frac{\partial^{2} \Theta(\cdot)}{\partial R_{k} \partial P_{\xi}} & \frac{\partial^{2} \Theta(\cdot)}{\partial P_{\xi} \partial O_{c r}} & \frac{\partial^{2} \Theta(\cdot)}{\partial R_{k}^{2}}
\end{array}\right|=\frac{\partial^{2} \Theta(\cdot)}{\partial P_{\xi}^{2}} \frac{\partial^{2} \Theta(\cdot)}{\partial O_{c r}^{2}} \frac{\partial^{2} \Theta(\cdot)}{\partial R_{k}^{2}}=\frac{b g}{P_{\xi}^{2} O_{c r}^{2}}\left(-\Gamma_{2}\right)=-\frac{\Gamma_{2} b g}{P_{\xi}^{2} O_{c r}^{2}}<0 .
\end{aligned}
$$




\section{Appendix E.}

$$
\begin{aligned}
& \left|H_{44}\right|=\left|\begin{array}{cccc}
\frac{\partial^{2} \Theta(\cdot)}{\partial P_{\xi}^{2}} & \frac{\partial^{2} \Theta(\cdot)}{\partial P_{\xi} \partial O_{c r}} & \frac{\partial^{2} \Theta(\cdot)}{\partial P_{\xi} \partial R_{k}} & \frac{\partial^{2} \Theta(\cdot)}{\partial P_{\xi} \partial L} \\
\frac{\partial^{2} \Theta(\cdot)}{\partial O_{c r} \partial P_{\xi}} & \frac{\partial^{2} \Theta(\cdot)}{\partial O_{c r}^{2}} & \frac{\partial^{2} \Theta(\cdot)}{\partial O_{c r} \partial R_{k}} & \frac{\partial^{2} \Theta(\cdot)}{\partial O_{c r} \partial L} \\
\frac{\partial^{2} \Theta(\cdot)}{\partial R_{k} \partial P_{\xi}} & \frac{\partial^{2} \Theta(\cdot)}{\partial R_{k} \partial O_{c r}} & \frac{\partial^{2} \Theta(\cdot)}{\partial R_{k}^{2}} & \frac{\partial^{2} \Theta(\cdot)}{\partial R_{k} \partial L} \\
\frac{\partial^{2} \Theta(\cdot)}{\partial P_{\xi} \partial L} & \frac{\partial^{2} \Theta(\cdot)}{\partial L \partial O_{c r}} & \frac{\partial^{2} \Theta(\cdot)}{\partial R_{k} \partial L} & \frac{\partial^{2} \Theta(\cdot)}{\partial L^{2}}
\end{array}\right|=\frac{\partial^{2} \Theta(\cdot)}{\partial P_{\xi}^{2}} \frac{\partial^{2} \Theta(\cdot)}{\partial O_{c r}^{2}}\left[\frac{\partial^{2} \Theta(\cdot)}{\partial R_{k}^{2}} \frac{\partial^{2} \Theta(\cdot)}{\partial L^{2}}-\left(\frac{\partial^{2} \Theta(\cdot)}{\partial R_{k} \partial L}\right)^{2}\right] \\
& =\frac{b g}{P_{\xi}^{2} O_{c r}^{2}}\left[\Gamma_{2} \Gamma_{3}-\Theta_{9}^{2}\right]>0 \text {, at } Q_{v}^{*}, i^{*}, L^{*}, p_{\mathrm{on}}^{*}, p_{\mathrm{off}}^{*}, P_{\xi}^{*}, O_{c r}^{*}, R_{k}^{*} \text {. }
\end{aligned}
$$

\section{Appendix F.}

$$
\begin{aligned}
\left|H_{55}\right| & =\left|\begin{array}{ccccc}
\frac{\partial^{2} \Theta(\cdot)}{\partial P_{\xi}^{2}} & \frac{\partial^{2} \Theta(\cdot)}{\partial P_{\xi} \partial O_{c r}} & \frac{\partial^{2} \Theta(\cdot)}{\partial P_{\xi} \partial R_{k}} & \frac{\partial^{2} \Theta(\cdot)}{\partial P_{\xi} \partial L} & \frac{\partial^{2} \Theta(\cdot)}{\partial P_{\xi} \partial p_{\text {on }}} \\
\frac{\partial^{2} \Theta(\cdot)}{\partial O_{c r} \partial P_{\xi}} & \frac{\partial^{2} \Theta(\cdot)}{\partial O_{c r}^{2}} & \frac{\partial^{2} \Theta(\cdot)}{\partial O_{c r} \partial R_{k}} & \frac{\partial^{2} \Theta(\cdot)}{\partial O_{c r} \partial L} & \frac{\partial^{2} \Theta(\cdot)}{\partial O_{c r} \partial p_{\text {on }}} \\
\frac{\partial^{2} \Theta(\cdot)}{\partial R_{k} \partial P_{\xi}} & \frac{\partial^{2} \Theta(\cdot)}{\partial R_{k} \partial O_{c r}} & \frac{\partial^{2} \Theta(\cdot)}{\partial R_{k}^{2}} & \frac{\partial^{2} \Theta(\cdot)}{\partial R_{k} \partial L} & \frac{\partial^{2} \Theta(\cdot)}{\partial R_{k} \partial p_{\text {on }}} \\
\frac{\partial^{2} \Theta(\cdot)}{\partial P_{\xi} \partial L} & \frac{\partial^{2} \Theta(\cdot)}{\partial L \partial O_{c r}} & \frac{\partial^{2} \Theta(\cdot)}{\partial R_{k} \partial L} & \frac{\partial^{2} \Theta(\cdot)}{\partial L^{2}} & \frac{\partial^{2} \Theta(\cdot)}{\partial L \partial p_{\mathrm{on}}} \\
\frac{\partial^{2} \Theta(\cdot)}{\partial P_{\xi} \partial p_{\mathrm{on}}} & \frac{\partial^{2} \Theta(\cdot)}{\partial p_{\text {on }} \partial O_{c r}} & \frac{\partial^{2} \Theta(\cdot)}{\partial R_{k} \partial p_{\mathrm{on}}} & \frac{\partial^{2} \Theta(\cdot)}{\partial L^{2}} & \frac{\partial^{2} \Theta(\cdot)}{\partial p_{\mathrm{on}}^{2}}
\end{array}\right| \\
= & -\frac{b g \Gamma_{4}}{P_{\xi}^{2} O_{c r}^{2}}\left(\Gamma_{2} \Gamma_{3}-\Theta_{9}^{2}\right)+\frac{g}{O_{c r}^{2}}\left(\frac{A_{f} i Q_{v} S_{c} \gamma p_{\mathrm{on}}^{-1}-\gamma}{2}\right)^{2}\left(\Gamma_{2} \Gamma_{3}-\Theta_{9}^{2}\right) .
\end{aligned}
$$

Therefore, $\left|H_{55}\right|<0$ at the optimum value of the decision variable i.e., at $\left(Q_{v}^{*}, i^{*}, L^{*}, p_{\mathrm{on}}^{*}, p_{\mathrm{off}}^{*}, P_{\xi}^{*}, O_{c r}^{*}, R_{k}^{*}\right)$.

\section{Appendix G.}

$$
\begin{aligned}
& \left|H_{66}\right|=\left|\begin{array}{cccccc}
\frac{\partial^{2} \Theta(\cdot)}{\partial P_{\xi}^{2}} & \frac{\partial^{2} \Theta(\cdot)}{\partial P_{\xi} \partial O_{c r}} & \frac{\partial^{2} \Theta(\cdot)}{\partial P_{\xi} \partial R_{k}} & \frac{\partial^{2} \Theta(\cdot)}{\partial P_{\xi} \partial L} & \frac{\partial^{2} \Theta(\cdot)}{\partial P_{\xi} \partial p_{\text {on }}} & \frac{\partial^{2} \Theta(\cdot)}{\partial P_{\xi} \partial p_{\text {off }}} \\
\frac{\partial^{2} \Theta(\cdot)}{\partial O_{c r} \partial P_{\xi}} & \frac{\partial^{2} \Theta(\cdot)}{\partial O_{c r}^{2}} & \frac{\partial^{2} \Theta(\cdot)}{\partial O_{c r} \partial R_{k}} & \frac{\partial^{2} \Theta(\cdot)}{\partial O_{c r} \partial L} & \frac{\partial^{2} \Theta(\cdot)}{\partial O_{c r} \partial p_{\text {on }}} & \frac{\partial^{2} \Theta(\cdot)}{\partial O_{c r} \partial p_{\text {off }}} \\
\frac{\partial^{2} \Theta(\cdot)}{\partial R_{k} \partial P_{\xi}} & \frac{\partial^{2} \Theta(\cdot)}{\partial R_{k} \partial O_{c r}} & \frac{\partial^{2} \Theta(\cdot)}{\partial R_{k}^{2}} & \frac{\partial^{2} \Theta(\cdot)}{\partial R_{k} \partial L} & \frac{\partial^{2} \Theta(\cdot)}{\partial R_{k} \partial p_{\text {on }}} & \frac{\partial^{2} \Theta(\cdot)}{\partial R_{k} \partial p_{\text {off }}} \\
\frac{\partial^{2} \Theta(\cdot)}{\partial P_{\xi} \partial L} & \frac{\partial^{2} \Theta(\cdot)}{\partial L \partial O_{c r}} & \frac{\partial^{2} \Theta(\cdot)}{\partial R_{k} \partial L} & \frac{\partial^{2} \Theta(\cdot)}{\partial L^{2}} & \frac{\partial^{2} \Theta(\cdot)}{\partial L \partial p_{\text {on }}} & \frac{\partial^{2} \Theta(\cdot)}{\partial L \partial p_{\text {off }}} \\
\frac{\partial^{2} \Theta(\cdot)}{\partial P_{\xi} \partial p_{\text {on }}} & \frac{\partial^{2} \Theta(\cdot)}{\partial p_{\text {on }} \partial O_{c r}} & \frac{\partial^{2} \Theta(\cdot)}{\partial R_{k} \partial p_{\text {on }}} & \frac{\partial^{2} \Theta(\cdot)}{\partial L \partial p_{\text {on }}} & \frac{\partial^{2} \Theta(\cdot)}{\partial p_{\text {on }}^{2}} & \frac{\partial^{2} \Theta(\cdot)}{\partial p_{\text {on }} \partial p_{\text {off }}} \\
\frac{\partial^{2} \Theta(\cdot)}{\partial P_{\xi} \partial p_{\text {off }}} & \frac{\partial^{2} \Theta(\cdot)}{\partial p_{\text {off }} \partial O_{c r}} & \frac{\partial^{2} \Theta(\cdot)}{\partial R_{k} \partial p_{\text {off }}} & \frac{\partial^{2} \Theta(\cdot)}{\partial L \partial p_{\text {off }}} & \frac{\partial^{2} \Theta(\cdot)}{\partial p_{\text {on }} \partial p_{\text {off }}} & \frac{\partial^{2} \Theta(\cdot)}{\partial p_{\text {off }}^{2}}
\end{array}\right| \\
& =-\frac{b}{P_{\xi}^{2}}\left|\begin{array}{ccccc}
\frac{\partial^{2} \Theta(\cdot)}{\partial O_{c r}^{2}} & \frac{\partial^{2} \Theta(\cdot)}{\partial O_{c r} \partial R_{k}} & \frac{\partial^{2} \Theta(\cdot)}{\partial O_{c r} \partial L} & \frac{\partial^{2} \Theta(\cdot)}{\partial O_{c r} \partial p_{\text {on }}} & \frac{\partial^{2} \Theta(\cdot)}{\partial O_{c r} \partial p_{\text {off }}} \\
\frac{\partial^{2} \Theta(\cdot)}{\partial R_{k} \partial O_{c r}} & \frac{\partial^{2} \Theta(\cdot)}{\partial R_{k}^{2}} & \frac{\partial^{2} \Theta(\cdot)}{\partial R_{k} \partial L} & \frac{\partial^{2} \Theta(\cdot)}{\partial R_{k} \partial p_{\text {on }}} & \frac{\partial^{2} \Theta(\cdot)}{\partial R_{k} \partial p_{\text {off }}} \\
\frac{\partial^{2} \Theta(\cdot)}{\partial L \partial O_{c r}} & \frac{\partial^{2} \Theta(\cdot)}{\partial R_{k} \partial L} & \frac{\partial^{2} \Theta(\cdot)}{\partial L^{2}} & \frac{\partial^{2} \Theta(\cdot)}{\partial L \partial p_{\text {on }}} & \frac{\partial^{2} \Theta(\cdot)}{\partial L \partial p_{\text {off }}} \\
\frac{\partial^{2} \Theta(\cdot)}{\partial p_{\text {on }} \partial O_{c r}} & \frac{\partial^{2} \Theta(\cdot)}{\partial R_{k} \partial p_{\text {on }}} & \frac{\partial^{2} \Theta(\cdot)}{\partial L \partial p_{\text {on }}} & \frac{\partial^{2} \Theta(\cdot)}{\partial p_{\text {on }}^{2}} & \frac{\partial^{2} \Theta(\cdot)}{\partial p_{\text {on }} \partial p_{\text {off }}} \\
\frac{\partial^{2} \Theta(\cdot)}{\partial p_{\text {off }} \partial O_{c r}} & \frac{\partial^{2} \Theta(\cdot)}{\partial R_{k} \partial p_{\text {off }}} & \frac{\partial^{2} \Theta(\cdot)}{\partial L \partial p_{\text {off }}} & \frac{\partial^{2} \Theta(\cdot)}{\partial p_{\text {on }} \partial p_{\text {off }}} & \frac{\partial^{2} \Theta(\cdot)}{\partial p_{\text {off }}^{2}}
\end{array}\right|
\end{aligned}
$$




$$
\begin{aligned}
& +\frac{A_{f} i Q_{v} S_{c} \gamma p_{\text {on }}^{-1-\gamma}}{2}\left|\begin{array}{ccccc}
\frac{\partial^{2} \Theta(\cdot)}{\partial O_{c r} \partial P_{\xi}} & \frac{\partial^{2} \Theta(\cdot)}{\partial O_{c r}^{2 r}} & \frac{\partial^{2} \Theta(\cdot)}{\partial O_{c r} \partial R_{k}} & \frac{\partial^{2} \Theta(\cdot)}{\partial O_{c r} \partial L} & \frac{\partial^{2} \Theta(\cdot)}{\partial O_{c} \partial p_{\text {off }}} \\
\frac{\partial^{2} \Theta(\cdot)}{\partial R_{k} \partial P_{\xi}} & \frac{\partial^{2} \Theta(\cdot)}{\partial R_{k} \partial O_{c r}} & \frac{\partial^{2} \Theta(\cdot)}{\partial R_{k}^{2}} & \frac{\partial^{2} \Theta(\cdot)}{\partial R_{k} \partial L} & \frac{\partial^{2} \Theta(\cdot)}{\partial R_{k} \partial p_{\text {off }}} \\
\frac{\partial^{2} \Theta(\cdot)}{\partial P_{\xi} \partial L} & \frac{\partial^{2} \Theta(\cdot)}{\partial L \partial O_{c r}} & \frac{\partial^{2} \Theta(\cdot)}{\partial R_{k} \partial L} & \frac{\partial^{2} \Theta(\cdot)}{\partial L^{2}} & \frac{\partial^{2} \Theta(\cdot)}{\partial L \partial p_{\text {off }}} \\
\frac{\partial^{2} \Theta(\cdot)}{\partial P_{\xi} \partial p_{\text {on }}} & \frac{\partial^{2} \Theta(\cdot)}{\partial p_{\text {on }} \partial O_{c r}} & \frac{\partial^{2} \Theta(\cdot)}{\partial R_{k} \partial p_{\text {on }}} & \frac{\partial^{2} \Theta(\cdot)}{\partial L \partial p_{\text {on }}} & \frac{\partial^{2} \Theta(\cdot)}{\partial p_{\text {on }} \partial p_{\text {off }}} \\
\frac{\partial^{2} \Theta(\cdot)}{\partial P_{\xi} \partial p_{\text {off }}} & \frac{\partial^{2} \Theta(\cdot)}{\partial p_{\text {off }} \partial O_{c r}} & \frac{\partial^{2} \Theta(\cdot)}{\partial R_{k} \partial p_{\text {off }}} & \frac{\partial^{2} \Theta(\cdot)}{\partial L \partial p_{\text {off }}} & \frac{\partial^{2} \Theta(\cdot)}{\partial p_{\text {off }}^{2}}
\end{array}\right| \\
& -\frac{\rho i Q_{v} S_{c} \delta p_{\text {off }}^{-1-\delta}}{2}\left|\begin{array}{ccccc}
\frac{\partial^{2} \Theta(\cdot)}{\partial O_{c r} \partial P_{\xi}} & \frac{\partial^{2} \Theta(\cdot)}{\partial O_{c r}^{2}} & \frac{\partial^{2} \Theta(\cdot)}{\partial O_{c r} \partial R_{k}} & \frac{\partial^{2} \Theta(\cdot)}{\partial O_{c r} \partial L} & \frac{\partial^{2} \Theta(\cdot)}{\partial O_{c r} \partial p_{\text {on }}} \\
\frac{\partial^{2} \Theta(\cdot)}{\partial R_{k} \partial P_{\xi}} & \frac{\partial^{2} \Theta(\cdot)}{\partial R_{k} \partial O_{c r}} & \frac{\partial^{2} \Theta(\cdot)}{\partial R_{k}^{2}} & \frac{\partial^{2} \Theta(\cdot)}{\partial R_{k} \partial L} & \frac{\partial^{2} \Theta(\cdot)}{\partial R_{k} \partial p_{\text {on }}} \\
\frac{\partial^{2} \Theta(\cdot)}{\partial P_{\xi} \partial L} & \frac{\partial^{2} \Theta(\cdot)}{\partial L \partial O_{c r}} & \frac{\partial^{2} \Theta(\cdot)}{\partial R_{k} \partial L} & \frac{\partial^{2} \Theta(\cdot)}{\partial L^{2}} & \frac{\partial^{2} \Theta(\cdot)}{\partial L \partial p_{\text {on }}} \\
\frac{\partial^{2} \Theta(\cdot)}{\partial P_{\xi} \partial p_{\text {on }}} & \frac{\partial^{2} \Theta(\cdot)}{\partial p_{\text {on }} \partial O_{c r}} & \frac{\partial^{2} \Theta(\cdot)}{\partial R_{k} \partial p_{\text {on }}} & \frac{\partial^{2} \Theta(\cdot)}{\partial L \partial p_{\text {on }}} & \frac{\partial^{2} \Theta(\cdot)}{\partial p_{\text {on }}^{2}} \\
\frac{\partial^{2} \Theta(\cdot)}{\partial P_{\xi} \partial p_{\text {off }}} & \frac{\partial^{2} \Theta(\cdot)}{\partial p_{\text {off }} \partial O_{c r}} & \frac{\partial^{2} \Theta(\cdot)}{\partial R_{k} \partial p_{\text {off }}} & \frac{\partial^{2} \Theta(\cdot)}{\partial L \partial p_{\text {off }}} & \frac{\partial^{2} \Theta(\cdot)}{\partial p_{\text {on }} \partial p_{\text {off }}}
\end{array}\right| .
\end{aligned}
$$

Therefore, $\left|H_{66}\right|>0$ at the optimum value of the decision variable i.e., at $\left(Q_{v}^{*}, i^{*}, L^{*}, p_{\mathrm{on}}^{*}, p_{\mathrm{off}}^{*}, P_{\xi}^{*}, O_{c r}^{*}, R_{k}^{*}\right)$.

\section{Appendix H.}

$$
\begin{aligned}
& \left|H_{77}\right|=\left|\begin{array}{ccccccc}
\frac{\partial^{2} \Theta(\cdot)}{\partial P_{\xi}^{2}} & \frac{\partial^{2} \Theta(\cdot)}{\partial P_{\xi} \partial O_{c r}} & \frac{\partial^{2} \Theta(\cdot)}{\partial P_{\xi} \partial R_{k}} & \frac{\partial^{2} \Theta(\cdot)}{\partial P_{\xi} \partial L} & \frac{\partial^{2} \Theta(\cdot)}{\partial P_{\xi} \partial p_{\text {on }}} & \frac{\partial^{2} \Theta(\cdot)}{\partial P_{\xi} \partial p_{\text {off }}} & \frac{\partial^{2} \Theta(\cdot)}{\partial P_{\xi} \partial Q_{v}} \\
\frac{\partial^{2} \Theta(\cdot)}{\partial O_{c r} \partial P_{\xi}} & \frac{\partial^{2} \Theta(\cdot)}{\partial O_{c r}^{2}} & \frac{\partial^{2} \Theta(\cdot)}{\partial O_{c r} \partial R_{k}} & \frac{\partial^{2} \Theta(\cdot)}{\partial O_{c r} \partial L} & \frac{\partial^{2} \Theta(\cdot)}{\partial O_{c r} \partial p_{\text {on }}} & \frac{\partial^{2} \Theta(\cdot)}{\partial O_{c r} \partial p_{\text {off }}} & \frac{\partial^{2} \Theta(\cdot)}{\partial O_{c r} \partial Q_{v}} \\
\frac{\partial^{2} \Theta(\cdot)}{\partial R_{k} \partial P_{\xi}} & \frac{\partial^{2} \Theta(\cdot)}{\partial R_{k} \partial O_{c r}} & \frac{\partial^{2} \Theta(\cdot)}{\partial R_{k}^{2}} & \frac{\partial^{2} \Theta(\cdot)}{\partial R_{k} \partial L} & \frac{\partial^{2} \Theta(\cdot)}{\partial R_{k} \partial p_{\text {on }}} & \frac{\partial^{2} \Theta(\cdot)}{\partial R_{k} \partial p_{\text {off }}} & \frac{\partial^{2} \Theta(\cdot)}{\partial R_{k} \partial Q_{v}} \\
\frac{\partial^{2} \Theta(\cdot)}{\partial P_{\xi} \partial L} & \frac{\partial^{2} \Theta(\cdot)}{\partial L \partial O_{c r}} & \frac{\partial^{2} \Theta(\cdot)}{\partial R_{k} \partial L} & \frac{\partial^{2} \Theta(\cdot)}{\partial L^{2}} & \frac{\partial^{2} \Theta(\cdot)}{\partial L \partial p_{\text {on }}} & \frac{\partial^{2} \Theta(\cdot)}{\partial L \partial p_{\text {off }}} & \frac{\partial^{2} \Theta(\cdot)}{\partial L \partial Q_{v}} \\
\frac{\partial^{2} \Theta(\cdot)}{\partial P_{\xi} \partial p_{\text {on }}} & \frac{\partial^{2} \Theta(\cdot)}{\partial p_{\text {on }} \partial O_{c r}} & \frac{\partial^{2} \Theta(\cdot)}{\partial R_{k} \partial p_{\text {on }}} & \frac{\partial^{2} \Theta(\cdot)}{\partial L \partial p_{\text {on }}} & \frac{\partial^{2} \Theta(\cdot)}{\partial p_{\text {on }}^{2}} & \frac{\partial^{2} \Theta(\cdot)}{\partial p_{\text {on }} \partial p_{\text {off }}} & \frac{\partial^{2} \Theta(\cdot)}{\partial p_{\text {on }} \partial Q_{v}} \\
\frac{\partial^{2} \Theta(\cdot)}{\partial P_{\xi} \partial p_{\text {off }}} & \frac{\partial^{2} \Theta(\cdot)}{\partial p_{\text {off }} \partial O_{c r}} & \frac{\partial^{2} \Theta(\cdot)}{\partial R_{k} \partial p_{\text {off }}} & \frac{\partial^{2} \Theta(\cdot)}{\partial L \partial p_{\text {off }}} & \frac{\partial^{2} \Theta(\cdot)}{\partial p_{\text {on }} \partial p_{\text {off }}} & \frac{\partial^{2} \Theta(\cdot)}{\partial p_{\text {off }}^{2}} & \frac{\partial^{2} \Theta(\cdot)}{\partial Q_{v} \partial p_{\text {off }}} \\
\frac{\partial^{2} \Theta(\cdot)}{\partial P_{\xi} \partial Q_{v}} & \frac{\partial^{2} \Theta(\cdot)}{\partial Q_{v} \partial O_{c r}} & \frac{\partial^{2} \Theta(\cdot)}{\partial R_{k} \partial Q_{v}} & \frac{\partial^{2} \Theta(\cdot)}{\partial L \partial Q_{v}} & \frac{\partial^{2} \Theta(\cdot)}{\partial p_{\text {on }} \partial Q_{v}} & \frac{\partial^{2} \Theta(\cdot)}{\partial Q_{v} \partial p_{\text {off }}} & \frac{\partial^{2} \Theta(\cdot)}{\partial Q_{v}^{2}}
\end{array}\right| \\
& =-\frac{b}{P_{\xi}^{2}}\left|\begin{array}{cccccc}
\frac{\partial^{2} \Theta(\cdot)}{\partial O_{c r}^{2}} & \frac{\partial^{2} \Theta(\cdot)}{\partial O_{c r} \partial R_{k}} & \frac{\partial^{2} \Theta(\cdot)}{\partial O_{c r} \partial L} & \frac{\partial^{2} \Theta(\cdot)}{\partial O_{c r} \partial p_{\text {on }}} & \frac{\partial^{2} \Theta(\cdot)}{\partial O_{c r} \partial p_{\text {off }}} & \frac{\partial^{2} \Theta(\cdot)}{\partial O_{c r} \partial Q_{v}} \\
\frac{\partial^{2} \Theta(\cdot)}{\partial R_{k} \partial O_{c r}} & \frac{\partial^{2} \Theta(\cdot)}{\partial R_{k}^{2}} & \frac{\partial^{2} \Theta(\cdot)}{\partial R_{k} \partial L} & \frac{\partial^{2} \Theta(\cdot)}{\partial R_{k} \partial p_{\text {on }}} & \frac{\partial^{2} \Theta(\cdot)}{\partial R_{k} \partial p_{\text {off }}} & \frac{\partial^{2} \Theta(\cdot)}{\partial R_{k} \partial Q_{v}} \\
\frac{\partial^{2} \Theta(\cdot)}{\partial L \partial O_{c r}} & \frac{\partial^{2} \Theta(\cdot)}{\partial R_{k} \partial L} & \frac{\partial^{2} \Theta(\cdot)}{\partial L^{2}} & \frac{\partial^{2} \Theta(\cdot)}{\partial L \partial p_{\text {on }}} & \frac{\partial^{2} \Theta(\cdot)}{\partial L \partial p_{\text {off }}} & \frac{\partial^{2} \Theta(\cdot)}{\partial L \partial Q_{v}} \\
\frac{\partial^{2} \Theta(\cdot)}{\partial p_{\text {on }} \partial O_{c r}} & \frac{\partial^{2} \Theta(\cdot)}{\partial R_{k} \partial p_{\text {on }}} & \frac{\partial^{2} \Theta(\cdot)}{\partial L \partial p_{\text {on }}} & \frac{\partial^{2} \Theta(\cdot)}{\partial p_{\text {on }}^{2}} & \frac{\partial^{2} \Theta(\cdot)}{\partial p_{\text {on }} \partial p_{\text {off }}} & \frac{\partial^{2} \Theta(\cdot)}{\partial p_{\text {on }} \partial Q_{v}} \\
\frac{\partial^{2} \Theta(\cdot)}{\partial p_{\text {off }} \partial O_{c r}} & \frac{\partial^{2} \Theta(\cdot)}{\partial R_{k} \partial p_{\text {off }}} & \frac{\partial^{2} \Theta(\cdot)}{\partial L \partial p_{\text {off }}} & \frac{\partial^{2} \Theta(\cdot)}{\partial p_{\text {on }} \partial p_{\text {off }}} & \frac{\partial^{2} \Theta(\cdot)}{\partial p_{\text {off }}^{2}} & \frac{\partial^{2} \Theta(\cdot)}{\partial Q_{v} \partial p_{\text {off }}} \\
\frac{\partial^{2} \Theta(\cdot)}{\partial Q_{v} \partial O_{c r}} & \frac{\partial^{2} \Theta(\cdot)}{\partial R_{k} \partial Q_{v}} & \frac{\partial^{2} \Theta(\cdot)}{\partial L \partial Q_{v}} & \frac{\partial^{2} \Theta(\cdot)}{\partial p_{\text {on }} \partial Q_{v}} & \frac{\partial^{2} \Theta(\cdot)}{\partial Q_{v} \partial p_{\text {off }}} & \frac{\partial^{2} \Theta(\cdot)}{\partial Q_{v}^{2}}
\end{array}\right|
\end{aligned}
$$




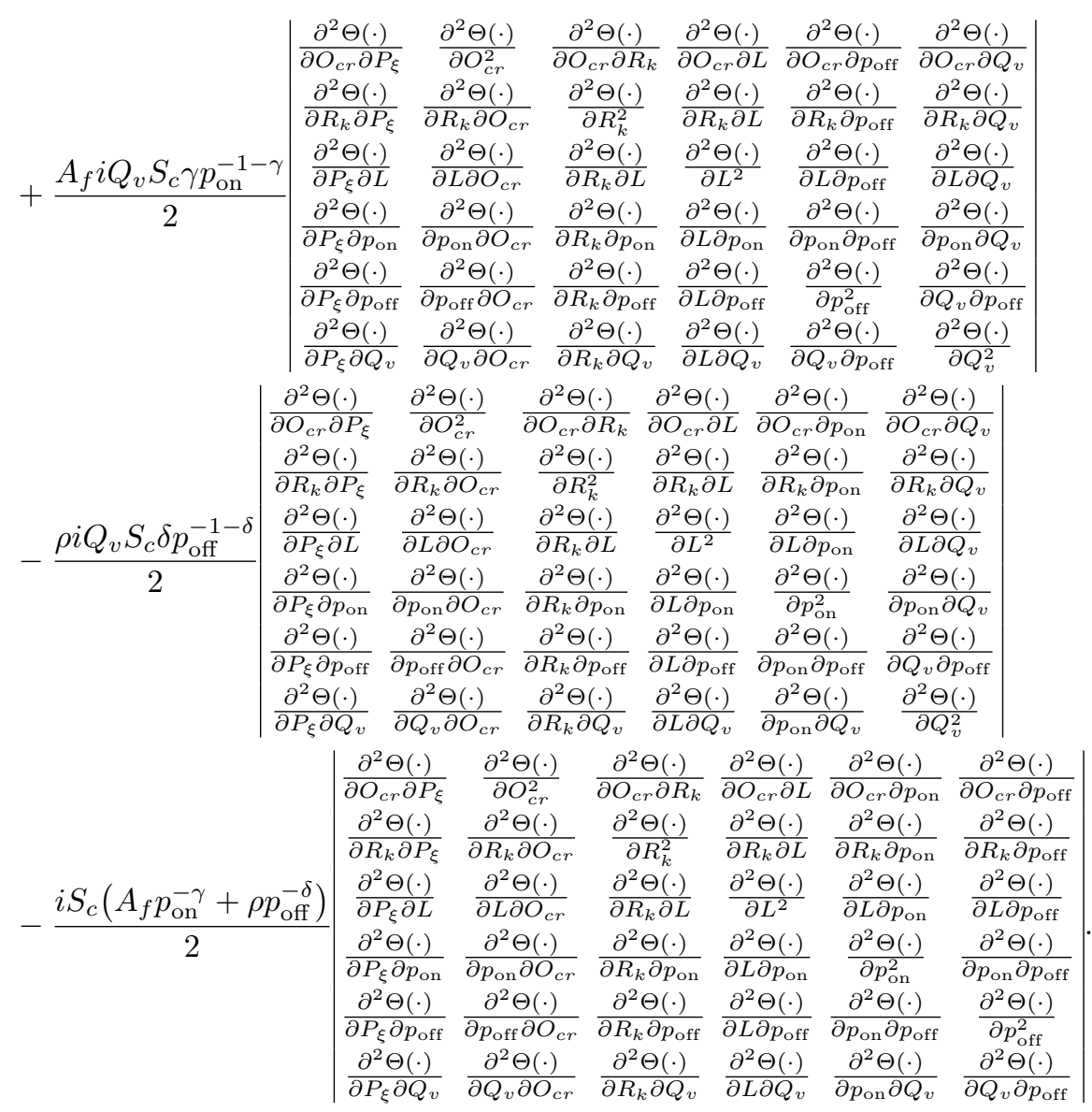

Therefore, $\left|H_{77}\right|<0$ at the optimum value of the decision variable i.e., at $\left(Q_{v}^{*}, i^{*}, L^{*}, p_{\mathrm{on}}^{*}, p_{\mathrm{off}}^{*}, P_{\xi}^{*}, O_{c r}^{*}, R_{k}^{*}\right)$.

Acknowledgements. This work was supported by Global Research Network program through the Ministry of Education of the Republic of Korea and the National Research Foundation of Korea (NRF-2017S1A2A2036758).

\section{REFERENCES}

[1] W. Ahmed, M. Moazzam, B. Sarkar and S.U. Rehman, Synergic effect of reworking for imperfect quality items with the integration of multi-period delay-in-payment and partial backordering in global supply chains. Engineering 7 (2021) 260-271.

[2] A. AlArjani, U.M. Modibbo, I. Ali and B. Sarkar, A new framework for the sustainable development goals of Saudi Arabia. J. King Saud Univ.-Sci. 33 (2021) 101477.

[3] L. Bao, Z. Liu, Y. Yu and W. Zhang, On the decomposition property for a dynamic inventory rationing problem with multiple demand classes and backorder. Eur. J. Oper. Res. 265 (2018) 99-106.

[4] M. Ben-Daya and A. Raouf, Inventory models involving lead time as a decision variable. J. Oper. Res. Soc. 45 (1994) $579-582$.

[5] S. Bhat and A. Krishnamurthy, Production control policies to maintain service levels in different seasons. J. Manuf. Syst. 41 (2016) 31-44.

[6] S. Bhuniya, S. Pareek and B. Sarkar, A supply chain model with service level constraints and strategies under uncertainty. Alexandria Eng. J. 60 (2021) 6035-6052.

[7] S. Bhuniya, S. Pareek, B. Sarkar and B.K. Sett, A smart production process for the optimum energy consumption with maintenance policy under a supply chain management. Processes $\mathbf{9}$ (2021) 19.

[8] X. Chao, B. Yang and Y. Xu, Dynamic inventory and pricing policy in a capacitated stochastic inventory system with fixed ordering cost. Oper. Res. Lett. 40 (2012) 99-107. 
[9] A. Chaudhuri, P.P. Datta, K.J. Fernandes and Y. Xiong, Optimal pricing strategies for manufacturing-as-a service platforms to ensure business sustainability. Int. J. Prod. Econ. 234 (2021) 108065.

[10] R. Clemons and S.A. Slotnick, The effect of supply-chain disruption, quality and knowledge transfer on firm strategy. Int. J. Prod. Econ. 178 (2016) 169-186.

[11] X.J. Dang, G.B. Bi, C.Y. Liu and Y. Xu, Optimal financing strategies with 3PL customized service in a capital-constrained supply chain. Electron. Commerce Res. App. 50 (2021) 101090.

[12] B.K. Dey, S. Bhuniya and B. Sarkar, Involvement of controllable lead time and variable demand for a smart manufacturing system under a supply chain management. Expert Syst. App. 184 (2021) 115464.

[13] B.K. Dey, S. Pareek, M. Tayyab and B. Sarkar, Autonomation policy to control work-in-process inventory in a smart production system. Int. J. Prod. Res. 59 (2021) 1258-1280.

[14] B.K. Dey, B. Sarkar and H. Seok, Cost-effective smart autonomation policy for a hybrid manufacturing-remanufacturing. Comput. Ind. Eng. 162 (2021) 107758.

[15] M. ElHafsi, J. Fang and E. Hamouda, Optimal production and inventory control of multi-class mixed backorder and lost sales demand class models. Eur. J. Oper. Res. 291 (2021) 147-161.

[16] A. Garai and B. Sarkar, Economically independent reverse logistics of customer-centric closed-loop supply chain for herbal medicines and biofuel. J. Cleaner Prod. 334 (2022) 129977.

[17] A. Garai, S. Chowdhury, B. Sarkar and T.K. Roy, Cost-effective subsidy policy for growers and biofuels-plants in closed-loop supply chain of herbs and herbal medicines: an interactive bi-objective optimization in T-environment. Appl. Soft Comput. $100(2021) 106949$.

[18] C.H. Glock, Lead time reduction strategies in a single-vendor-single-buyer integrated inventory model with lot size-dependent lead times and stochastic demand. Int. J. Prod. Econ. 136 (2012) 37-44.

[19] K. Govindan and A. Malomfalean, A framework for evaluation of supply chain coordination by contracts under O2O environment. Int. J. Prod. Econ. 215 (2019) 11-23.

[20] K. Govindan, S.G. Azevedo, H. Carvalho and V. Cruz-Machado, Impact of supply chain management practices on sustainability. J. Cleaner Prod. 85 (2014) 212-225.

[21] R. Guchhait and B. Sarkar, Economic and environmental assessment of an unreliable supply chain management. RAIRO: OR 55 (2021) 3153-3170.

[22] M.S. Habib, O. Asghar, A. Hussain, M. Imran, M.P. Mughal and B. Sarkar, A robust possibilistic programming approach toward animal fat-based biodiesel supply chain network design under uncertain environment. J. Cleaner Prod. 278 (2021) 122403.

[23] Z. He, T. Cheng, J. Dong and S. Wang, Evolutionary location and pricing strategies for service merchants in competitive O2O markets. Eur. J. Oper. Res. 254 (2016) 595-609.

[24] B. He, P. Mirchandani and Y. Wang, Removing barriers for grocery stores: O2O platform and self-scheduling delivery capacity. Transp. Res. Part E: Logistics Transp. Rev. 141 (2020) 102036.

[25] J. Heydari, P. Zaabi-Ahmadi and T.M. Choi, Coordinating supply chains with stochastic demand by crashing lead times. Comput. Oper. Res. 100 (2018) 394-403.

[26] J. Ji, Z. Zhang and L. Yang, Comparisons of initial carbon allowance allocation rules in an O2O retail supply chain with the cap-and-trade regulation. Int. J. Prod. Econ. 187 (2017) 68-84.

[27] B. Jiang and B. Yang, Quality and pricing decisions in a market with consumer information sharing. Manage. Sci. 65 (2019) $272-285$.

[28] H. Jodlbauer and S. Reitner, Optimizing service-level and relevant cost for a stochastic multi-item cyclic production system. Int. J. Prod. Econ. 136 (2012) 306-317.

[29] M. Khan, M. Hussain and L.E. Cárdenas-Barrón, Learning and screening errors in an EPQ inventory model for supply chains with stochastic lead time demands. Int. J. Prod. Res. 55 (2017) 4816-4832.

[30] R. Kumar, R.K. Chandrawat, B. Sarkar, V. Joshi and A. Majumder, An advanced optimization technique for smart production using $\alpha$-cut based quadrilateral fuzzy number. Int. J. Fuzzy Syst. 23 (2021) 107-127.

[31] B. Khurshid, S. Maqsood, M. Omair, B. Sarkar, M. Saad and U. Asad, Fast evolutionary algorithm for flow shop scheduling problems. IEEE Access. 9 (2021) 44825-44839.

[32] W.C. Lee, J.W. Wu and C.L. Lei, Computational algorithmic procedure for optimal inventory policy involving ordering cost reduction and back-order discounts when lead time demand is controllable. Appl. Math. Comput. 189 (2007) 186-200.

[33] X. Li, Valuing lead-time and its variance in batch-ordering inventory policies. Int. J. Prod. Econ. 228 (2020) 107731.

[34] X. Li, Y. Li and W. Cao, Cooperative advertising models in O2O supply chains. Int. J. Prod. Econ. 215 (2019) $144-152$.

[35] K.R. Lou and W.C. Wang, A comprehensive extension of an integrated inventory model with ordering cost reduction and permissible delay in payments. Appl. Math. Model. 37 (2013) 4709-4716.

[36] D. Ma, J. Hu and F. Yao, Big data empowering low-carbon smart tourism study on low-carbon tourism O2O supply chain considering consumer behaviors and corporate altruistic preferences. Comput. Ind. Eng. 153 (2021) 107061.

[37] A.S. Mahapatra, H.N. Soni, M.S. Mahapatra, B. Sarkar and S. Majumder, A continuous review production-inventory system with a variable preparation time in a fuzzy random environment. Mathematics 9 (2021) 747.

[38] A.H.M. Mashud and B. Sarkar, Retailer's joint pricing model through an effective preservation strategy under a trade-credit policy. RAIRO: OR $\mathbf{5 5}$ (2021) 1799.

[39] U. Mishra, J.Z. Wu and B. Sarkar, Optimum sustainable inventory management with backorder and deterioration under controllable carbon emissions. J. Cleaner Prod. 279 (2021) 123699. 
[40] R. Nandra, K.R. Marak, R. Kaur, B.K. Dey and A. Majumder, Establishing relation between production rate and product quality in a single-vendor multi-buyer supply chain model. Int. J. Serv. Oper. Inf. 11 (2021) 315-331.

[41] R. Nandra, A. Majumder and M. Mishra, A multi-retailer sustainable supply chain model with information sharing and quality deterioration. RAIRO: OR $\mathbf{5 5}$ (2021) S2773-S2794.

[42] M. Noori-Daryan, A.A. Taleizadeh and F. Jolai, Analyzing pricing, promised delivery lead time, supplier-selection, and ordering decisions of a multi-national supply chain under uncertain environment. Int. J. Prod. Econ. 209 (2019) $236-248$.

[43] D. Panda, M.K. Maiti and M. Maiti, Two warehouse inventory models for single vendor multiple retailers with price and stock dependent demand. Appl. Math. Modell. 34 (2010) 3571-3585.

[44] V. Pando, L.A. San-José, J. Sicilia and D. Alcaide-Lopez-de Pablo, Maximization of the return on inventory management expense in a system with price-and stock-dependent demand rate. Comput. Oper. Res. 127 (2021) 105134.

[45] Z. Pei, B.R. Wooldridge and K.R. Swimberghe, Manufacturer rebate and channel coordination in O2O retailing. J. Retailing Consum. Serv. 58 (2021) 102268.

[46] S. Saha, D. Chatterjee and B. Sarkar, The ramification of dynamic investment on the promotion and preservation technology for inventory management through a modified flower pollination algorithm. J. Retailing Consum. Serv. 58 (2021) 102326.

[47] S. Samanta, V.K. Dubey and B. Sarkar, Measure of influences in social networks. Appl. Soft Comput. 99 (2021) 106858.

[48] S.S. Sana, Price competition between green and non green products under corporate social responsible firm. J. Retailing Consum. Serv. 55 (2020) 102118.

[49] S.S. Sana and K. Chaudhuri, A deterministic EOQ model with delays in payments and price-discount offers. Eur. J. Oper. Res. 184 (2008) 509-533.

[50] M. Sarkar and B.D. Chung, Flexible work-in-process production system in supply chain management under quality improvement. Int. J. Prod. Res. 58 (2020) 3821-3838.

[51] B. Sarkar, M. Ullah and S.B. Choi, Joint inventory and pricing policy for an online to offline closed-loop supply chain model with random defective rate and returnable transport items. Mathematics 7 (2019) 497.

[52] B. Sarkar, B.K. Dey, M. Sarkar and A. AlArjani, A sustainable online-to-offline (O2O) retailing strategy for a supply chain management under controllable lead time and variable demand. Sustainability 13 (2021) 1756.

[53] B. Sarkar, M. Ullah and M. Sarkar, Environmental and economic sustainability through innovative green products by remanufacturing. J. Cleaner Prod. 332 (2022) 129813.

[54] B. Sarkar, B. Mridha and S. Pareek, A sustainable smart multi-type biofuel manufacturing with the optimum energy utilization under flexible production. J. Cleaner Prod. 332 (2022) 129869.

[55] B. Sarkar, A. Debnath, A.S. Chiu and W. Ahmed, Circular economy-driven two-stage supply chain management for nullifying waste. J. Cleaner Prod. 339 (2022) 130513.

[56] A. Sepehri, U. Mishra, M.L. Tseng and B. Sarkar, Joint pricing and inventory model for deteriorating items with maximum lifetime and controllable carbon emissions under permissible delay in payments. Mathematics 9 (2021) 470.

[57] A. Sepehri, U. Mishra and B. Sarkar, A sustainable production-inventory model with imperfect quality under preservation technology and quality improvement investment. J. Cleaner Prod. 310 (2021) 127332.

[58] B.K. Sett, B.K. Dey and B. Sarkar, The effect of O2O retail service quality in supply chain management. Mathematics 8 (2020) 1743.

[59] S.R. Singh, D. Yadav, B. Sarkar and M. Sarkar, Impact of energy and carbon emission of a supply chain management with two-level trade-credit policy. Energies 14 (2021) 1569.

[60] A. Srivastav and S. Agrawal, Multi-objective optimization of hybrid backorder inventory model. Expert Syst. App. 51 (2016) 76-84.

[61] Z. Xu, H. Zhang, J. Zhang and R.Q. Zhang, Online demand fulfillment under limited flexibility. Manage. Sci. 66 (2020) $4667-4685$.

\section{Subscribe to Open (S20)}

\section{A fair and sustainable open access model}

This journal is currently published in open access under a Subscribe-to-Open model (S2O). S2O is a transformative model that aims to move subscription journals to open access. Open access is the free, immediate, online availability of research articles combined with the rights to use these articles fully in the digital environment. We are thankful to our subscribers and sponsors for making it possible to publish this journal in open access, free of charge for authors.

\section{Please help to maintain this journal in open access!}

Check that your library subscribes to the journal, or make a personal donation to the $\mathrm{S} 2 \mathrm{O}$ programme, by contacting subscribers@edpsciences.org

More information, including a list of sponsors and a financial transparency report, available at: https://www. edpsciences.org/en/maths-s2o-programme 